\title{
Learning and Complementarities: Implications for Speculative Attacks*
}

\author{
Itay Goldstein, Emre Ozdenoren, and Kathy Yuan ${ }^{\dagger}$
}

November 2007

\begin{abstract}
We study a model where the aggregate trading of currency speculators reveals new information to the central bank and affects its decision on whether to abandon a fixed exchange rate regime. We show that the learning process gives rise to informational complementarities among traders, which generates coordination motives in their trading and reduces the informational content of the market signal and the efficiency of the central bank's decision. We analyze the tradeoff between information and coordination and derive comparative statics regarding the efficiency of the central bank's decision. We show that by committing to overweight its own beliefs, the central bank can improve the ex ante efficiency of its decisions. We also find that transparency may reduce the ability of the central bank to learn from the aggregate action of the speculators. Overall, our results apply to a wide range of problems, where decision makers learn from the actions of heterogeneously informed agents.
\end{abstract}

Journal of Economic Literature Classification Codes: G12, G15, F39, C7.

Keywords: Currency attacks, financial markets, global games, strategic complementarities, heterogenous information, learning, coordination.

*We thank Franklin Allen, Rossella Argenziano, Sohnke Bartam, Tilman Börgers, Amil Dasgupta, Joao Gomes, Han Kim, Gregor Matvos, Stephen Morris, Alessandro Pavan, Lones Smith, Avindhar Subrahmanyam, and seminar participants at Boston College, the International Finance Conference at Queen's University, Johns Hopkins University, the Stony Brook Workshop on Global Games, University of Michigan, University of Pennsylvania, and University of Toronto for their many insightful comments.

${ }^{\dagger}$ Goldstein is from the Department of Finance, Wharton School, University of Pennsylvania. E-mail: itayg@wharton.upenn.edu. Ozdenoren is from the Department of Economics, University of Michigan. Email: emreo@umich.edu. Yuan is from the Department of Finance, Ross School, University of Michigan. E-mail: kyuan@umich.edu 


\section{Introduction}

Many decision makers inspect the events in financial markets to gather information. Firm managers learn from the market about the profitability of proposed investments and use this information when making real investment decisions (Luo (2005) and Chen, Goldstein, and Jiang (2007)). Providers of capital use the information in the market to decide whether to finance investment projects (Baker, Stein, and Wurgler (2003)). Policymakers use market information when setting interest rates and making other decisions (Piazzesi (2005)). Financial markets are known to provide useful information because they aggregate opinions from many different speculators, who trade on their own money and are too numerous to be communicated with outside the trading process. Roll (1984) and Wolfers and Zitzewitz (2004) provide evidence that demonstrate the accuracy of financial-market information.

We show that when using the information aggregated in the market, decision makers adversely affect the trading incentives of speculators in a way that reduces the informational content of the market signal. Hence, learning from market events can be self-defeating. In our model, since the aggregate action of speculators affects a decision that changes the value of the traded security, strategic complementarities arise among speculators and make them want to trade like others. As a result, the signal that comes from the aggregate action of speculators reflects not only information about asset fundamentals, but also coordination among speculators. We model these forces and analyze the equilibrium outcomes in the context of a central bank that tries to learn from the size of a speculative attack about the optimal exchange rate regime.

Speculative attacks in currency markets lead central banks to change their exchange rate policies and even to abandon a fixed exchange rate regime. Existing models of speculative attacks offer one common mechanism that centers on the fact that a large speculative attack causes a substantial loss of reserves (see Salant and Henderson (1978), Flood and Garber (1984), and Krugman (1979) for first-generation models, and Obstfeld (1996) and Morris and Shin (1998) for second-generation models). In our model, speculative attacks affect the central bank's decision because of the information they contain. In particular, we study a situation where the central bank is only partially informed about the fundamentals of the economy. Speculators in the currency market also have pieces of information that guide their decision whether to join a speculative attack. By observing the aggregate size of the attack, the central bank gets an aggregate picture of the pieces of information held by speculators. 
Seeing a large attack against the currency, the central bank may come to believe that the fundamentals are bad and thus that it should abandon the regime. ${ }^{1}$

In the specific context of speculative attacks, the motivation for this approach is twofold. First, the relevance of the traditional approach in capturing the real-world environment has decreased over time. Losing reserves does not seem to be as costly as postulated by the traditional approach. This is because central banks, especially those in the developed countries, often have access to large short-term borrowing facilities for foreign reserves from other central banks or multilateral agencies. In addition, other defensive mechanisms such as raising short-term interest rates or implementing differential credit controls and restrictions on currency speculators also are available. ${ }^{2}$ Finally, the traditional approach is suited to explain only speculative attacks against an over-appreciated currency, since reserves do not pose a problem when the currency is over-depreciated. Our approach can be used to analyze attacks against an over-depreciated currency, such as the Chinese Yuan.

Second, the idea that the central bank is not fully informed about the state of the economy and that it can gain additional information by observing market outcomes is very natural. Trading in the currency market aggregates private information of speculators. This private information can simply be speculators' private interpretation of public news about various aspects of the economy. It also can be information that is not publicly observed. For example, take the model of Burnside, Eichenbaum, and Rebelo (2001). They argue that prospective deficits associated with implicit bailout guarantees to failing banks were the reason for the crises. Relating this to our paper, one can argue that the currency market aggregates market participants' heterogeneous understanding of the severity of the impact of implicit bailout guarantees. Indeed, existing empirical literature documents the informational content of currency trading. ${ }^{3}$ Moreover, in a recent paper, Piazzesi (2005) documents that in the US, the Federal Reserve Bank is affected by market developments when setting monetary policy. Finally, it is important to note that the notion that central banks are fully informed before a speculative attack erupts seems inconsistent with anecdotal

\footnotetext{
${ }^{1}$ The regime change decision in the model can be interpreted more broadly to capture other central bank policy decisions such as intervention in a managed-float exchange rate environment.

${ }^{2}$ Drazen (2000) has a detailed description of these defensive tools.

${ }^{3}$ For example, Ito, Lyons, and Melvin (1998) show that foreign exchange trading contains private information; Lyons (2001) and Evans and Lyons (2002) show that order flows forecast changes in exchange rates; and Danielsson and Saltoglu (2003) show that institutional order flows are informative about exchange rate changes.
} 
evidence from real-world episodes. In these episodes, it is often the case that a central bank defends the currency regime for a period of time, and then abandons it. Such behavior can be rationalized only if the central bank learns something during the course of the attack. ${ }^{4}$

Our analysis shows that, by using the information captured in the size of the speculative attack, the central bank is able to improve the efficiency of its decision on whether to maintain the exchange rate regime. Yet, learning from the currency market comes with a cost. Assuming that there is some commonality in the pieces of information observed by market participants, we find that market participants put excessive weight on the common information when deciding whether to participate in a speculative attack. As a result, the aggregate attack reflects both information and coordination, and the efficiency of the central bank's policy decision is reduced. Hence, the information aggregated in the process of a speculative attack provides a double-edged sword to the central bank.

The reason behind this result is that when the central bank learns from the aggregate action of multiple speculators, strategic complementarities emerge among them. That is, speculators have an incentive to do what other speculators do, so they put excessive weight on the common signal, which is informative not only about the fundamentals but also about the action of other speculators. Thus, speculative attacks in our model have some similarity to those in Morris and Shin (1998) and other related papers in that they come as a result of a coordinated action among speculators, and they lead the central bank to take an inefficient action. Importantly, however, unlike in other papers where strategic complementarities are assumed to exist, in this paper they emerge endogenously as an inherent feature of information aggregation and learning. We thus refer to the complementarities in our paper as informational complementarities.

What causes informational complementarities to emerge? The idea is that if many speculators are going to attack even when the fundamentals are not bad, then the aggregate attack may fool the central bank to think that the fundamentals are bad and to abandon the regime. Knowing this, it is optimal for each individual speculator to join the attack as well. Thus, informational complementarities come solely due to the fact that the central bank is learning from the aggregate action of speculators. ${ }^{5}$ The irony is that by learning from the

\footnotetext{
${ }^{4}$ Angeletos, Hellwig, and Pavan (2007) consider learning in the other direction. They consider a dynamic model where speculators learn about the fundamental by observing the actions of the central bank over time. Angeletos and Werning (2006) and Hellwig, Mukherji, and Tsyvinski (2006) study learning among speculators when they all observe prices.

${ }^{5}$ Note that the informational complementarities here are different from the complementarities in the
} 
aggregate action of speculators the central bank reduces the informational content in this action. Morris and Shin (2005) note that the central bank is an active shaper of events and an observer of events at the same time. Our model demonstrates that these two roles interfere with each other.

Our analysis shows that the informational complementarities considered in our paper generate quite different implications from the usual direct complementarities (as in Morris and Shin (1998)). For example, while a decrease in the transaction cost of attacking the currency increases the probability that speculators will participate in a speculative attack, it has no effect on the probability that the central bank will abandon the regime. This is because the central bank cares only about the informational content of the attack, and thus filters out the effect that transaction costs have on its size. This may be an explanation to the empirical puzzle that some speculative attacks are defended but some, equally strong, are not (Rebelo (2000)).

Based on this logic, variables that characterize the quality of information turn out to have the most substantial effect in our model. We are particularly interested in the effect of these variables on the efficiency of the central bank's policy decision. Quite expectably, we find that the quality of the central bank's private information increases the efficiency of its policy decision. On the other hand, the effect of the quality of the speculators' information turns out to be more complex. When the idiosyncratic elements of their information sets become more precise, the central bank is more likely to take the efficient decision. This is because speculators' information gets transmitted via the trading process and guides the central bank in its policy decision. But the precision of the common element in the speculators' information sets may have the opposite effect: When the common signal held by speculators gets more precise, their ability to coordinate on misleading the central bank into taking the wrong action improves. This result is reminiscent of Morris and Shin (2002), where an improvement in the precision of a public signal can reduce efficiency. The stark difference, however, is that in their model there are assumed strategic complementarities that lead to this result, whereas here strategic complementarities emerge endogenously due to learning. Overall, given the importance of the informativeness of the trading process, potential tests of decision to produce information studied in papers like Froot, Scharfstein, and Stein (1992); Hirshleifer, Subrahmanyam, and Titman (1994); Bru and Vives (2002); Veldkamp (2006a and 2006b); and Dow, Goldstein, and Guembel (2007). Here, speculators want to trade in the same direction as others, while in the other papers they want to produce information if other speculators produce information. 
our model can be based on market microstructure measures as in Easley and O'Hara (1987).

We also study the desirability of two policy tools in our framework. First, we show that the central bank can improve the ex ante efficiency of its policy decision by committing to put a lower weight on the information conveyed by the speculative attack than is expost efficient. While this generates ex-post inefficiency, it changes the ex ante incentives of speculators in a way that makes them put a lower weight on their common information. This, in turn, increases the informativeness of their aggregate action. Second, we analyze the effects of central bank transparency. ${ }^{6}$ We find that the impact of transparency is quite delicate. We discuss two related notions of transparency. In the first, speculators commonly observe a noisy signal of the central bank's information. We show that this reduces efficiency because it provides common information to the speculators about the action that the central bank is likely to take, and this enables the speculators to coordinate on their common information more effectively. In the second notion, speculators heterogeneously interpret the central bank's communication. In this case, more transparency is always good, because giving speculators better heterogeneous information reduces their ability to coordinate and leads them to reveal more accurate information through the attack.

As emphasized in the beginning of the paper, our model applies to a wide range of problems, where decision makers learn from the actions of heterogeneously informed agents. In this, our paper belongs to the growing literature on the feedback effect from trading in financial markets to the real economy. Earlier papers by Fishman and Hagerty (1992); Leland (1992); Khanna, Slezak, and Bradley (1994); Boot and Thakor (1997); Dow and Gorton (1997); Subrahmanyam and Titman (1999); and Fulghieri and Lukin (2001) analyze models where the information revealed in the trading process affects decisions in the real economy. Our paper brings a new angle to this literature. It shows that the fact that a decision maker in the real side of the economy learns from the trading process may generate strategic complementarities among traders and lead to equilibria where they act as if they coordinate to manipulate the beliefs of the decision maker. Our paper is closely related to two recent papers in this literature. Ozdenoren and Yuan (2007) show that the feedback effect from asset prices to the real value of a firm generates strategic complementarities. In their paper, however, the feedback effect is modeled exogenously and is not based on learning. Goldstein

\footnotetext{
${ }^{6}$ There is a large literature on transparency. Some recent works include Morris and Shin (2002, 2005), Heinemann and Cornand (2004), Woodford (2005), Svensson (2005), Hellwig (2005), and Angeletos and Pavan (2007).
} 
and Guembel (2007) do analyze learning by a decision maker, and show that this might lead to manipulation of the price by a single potentially informed trader. So the manipulation equilibrium is not a result of strategic complementarities among heterogeneously informed traders. $^{7}$

Finally, in a concurrent and independent paper, Angeletos, Lorenzoni, and Pavan (2007) analyze how learning by Wall Street traders from aggregate investments of "Silicon Valley" firms can lead to endogenous complementarities. Our paper is different from theirs in the context of the study and in the modeling device. Hence, the two papers yield different results and implications.

The remainder of this paper is organized as follows. In Section 2, we present the model setup. Section 3 characterizes the equilibrium of the model. In Section 4, we describe the notion of informational complementarities that emerges in our model. Section 5 analyzes the implications of the model for the efficiency of the central bank's decision on whether to abandon the exchange rate regime. In Section 6, we analyze the desirability of two policy tools in our model. Section 7 concludes.

\section{The Model Setup}

The players in our model are a central bank and a continuum of currency speculators. Initially, there is a currency peg in place. The central bank has to make a decision whether to maintain the currency peg or not. The value of maintaining the currency peg is characterized by a random state of the fundamental $\theta$. The regime outcome is given by $\delta \in\{0,1\}$ where $\delta=1$ indicates that the central bank defends the status quo and $\delta=0$ indicates that the central bank abandons the status quo. The regime outcome $\delta$ is controlled by the central bank. The central bank's payoff is given by

$$
U=\delta \theta .
$$

The central bank optimally chooses the regime $\delta$ to maximize $U$. When the central bank is perfectly informed about the fundamental, the optimal decision is to simply set $\delta=1$ if $\theta>0$ and set $\delta=0$ if $\theta<0$. In reality, the central bank is likely to be imperfectly informed, and our analysis focuses on the central bank's exchange rate policy in this case.

\footnotetext{
${ }^{7}$ See also Khanna and Sonti (2004), where manipulation happens as a result of the feedback effect. In their paper, feedback is exogenous and not based on learning.
} 
A continuum of speculators of measure one, indexed by $i$ and uniformly distributed over $[0,1]$, decide whether to attack (i.e., take an action that favors regime change) or abstain from attacking (i.e., take an action that favors the status quo). We assume that speculators are wealth constrained and can only short-sell up to one unit of the currency. The opportunity cost of attacking, i.e., short-selling one unit of the currency, is given by $c \in(0,1)$. The payoff of a speculator who does not attack is normalized to zero. The payoff from attacking the currency is $1-c$ if the status quo is abandoned (i.e., if the central bank sets $\delta=0$ ) and $-c$ otherwise.

The central bank and the speculators play the following game. First, both the central bank and the speculators receive information regarding $\theta$. Then the speculators independently and simultaneously decide whether to attack the currency or not. Finally, after observing the size of the aggregate attack from speculators, the central bank decides whether to maintain the status quo or not.

We assume that the central bank and the speculators have a common prior about $\theta$ which is an improper uniform over $\mathbb{R}$, and the central bank receives a private signal $s_{c}=\theta+\sigma_{c} \epsilon_{c}$ about the fundamental, where $\epsilon_{c}$ is normally distributed with mean of zero and standard deviation one. We denote the p.d.f. and the c.d.f. of the standard normal distribution by $\phi$ and $\Phi$ respectively. We assume that the central bank observes the size of the attack from informed speculators denoted by $A$. Equivalently, the central bank observes $T=\Phi^{-1}(A)$, which we use in the rest of the paper to simplify the exposition. We assume that speculator $i \in[0,1]$ receives a signal $s_{i}=\theta+\sigma_{s} \epsilon_{i}$ about the fundamental, where $\epsilon_{i}$ is normally distributed with a mean of zero and a standard deviation of one. Notice that we are not assuming that speculators individually are better informed than the central bank. In fact, each speculator's information might be much noisier than the central bank's information.

Finally, we assume that speculators receive a common noisy signal about the fundamental, in addition to their private signals. We denote the common signal by $s_{p}=\theta+\sigma_{p} \epsilon_{p}$ where $\epsilon_{p}$ is normally distributed with a mean of zero and a standard deviation of one. We assume that this signal is not observed by the central bank. (We will elaborate on the role of this assumption and different possible justifications in Section 4.) The payoff and information structure are common knowledge. All error terms $-\epsilon_{c}, \epsilon_{p}$, and $\epsilon_{i}-$ are independent of each other; the $\epsilon_{i}$ terms are also independent across investors.

Before we proceed with solving the model, a few comments about the setup are in order. First, the value to maintaining the currency regime, $\theta$, in our model, may be interpreted 
as related to the economic and political fundamentals of the economy, consistent with the level of the economy's sustainable exchange rate. Second, in our model, the central bank does not face an exogenously given cost in defending the currency regime. Specifically, the size of the speculative attack does not enter the central bank's payoff function in Equation (1). By comparison, the existing literature assumes that the cost of defending the currency regime is increasing in the size of the currency attack, and hence strategic complementarities among speculators in attacking the fixed exchange rate regime are exogenously given. We demonstrate in the later sections that even though there are no such built-in strategic complementarities in our model, a speculator may prefer to attack when others are attacking in equilibrium. That is, strategic complementarities may arise endogenously. Third and finally, while the existing literature assumes that the central bank knows the state of the fundamentals, $\theta$, perfectly, we assume that the central bank observes $\theta$ imperfectly, which is empirically relevant. In our model, currency trading aggregates information. The central bank, in turn, does not ignore the aggregate information revealed in the speculative attack and takes it into consideration in making currency policy decisions.

\section{Equilibrium}

We now formally define an equilibrium in our model. Let $g\left(s_{i}, s_{p}\right)$ denote the action of a speculator given private signal $s_{i}$ and common signal $s_{p}, T\left(\theta, s_{p}\right)$ the size of the aggregate attack from speculators for given fundamental $\theta$ and common signal $s_{p}$, and $\delta\left(T, s_{c}\right)$ the action of the central bank as a function of the size of the attack and its signal. ${ }^{8}$ Furthermore, let $\nu\left(\theta \mid T, s_{c}\right)$ denote the posterior distribution of $\theta$ conditional on the central bank's information, and let $\mu\left(\theta \mid s_{i}, s_{p}\right)$ denote the posterior distribution of $\theta$ conditional on a speculator's information.

Definition 1 An equilibrium consists of a strategy for the central bank, $\delta\left(T, s_{c}\right)$, a symmetric

\footnotetext{
${ }^{8}$ Recall that $T=\Phi^{-1}(A)$ where $A$ is the realized size of the attack.
} 
strategy for the agents, $g\left(s_{i}, s_{p}\right)$, probability measures, $\nu\left(\cdot \mid T, s_{c}\right)$ and $\mu\left(\cdot \mid s_{i}, s_{p}\right)$, such that

$$
\begin{aligned}
& \delta\left(T, s_{c}\right) \in \operatorname{argmax}_{\delta \in\{0,1\}} \int_{-\infty}^{\infty} \delta \theta d \nu\left(\theta \mid T, s_{c}\right), \\
& g\left(s_{i}, s_{p}\right) \in \operatorname{argmax}_{a \in\{0,1\}} a \cdot\left[\int_{-\infty}^{\infty} \int_{-\infty}^{\infty} \mathbf{1}_{\left[\delta\left(T\left(\theta, s_{p}\right), \theta+\sigma_{c} \epsilon_{c}\right)=0\right]} d \mu\left(\theta \mid s_{i}, s_{p}\right) d \Phi\left(\epsilon_{c}\right)-c\right], \\
& T\left(\theta, s_{p}\right)=\Phi^{-1}\left(\int_{-\infty}^{\infty} g\left(\theta+\sigma_{s} \epsilon, s_{p}\right) \phi(\epsilon) d \epsilon\right), \\
& \nu\left(\theta \mid T, s_{c}\right) \text { is obtained using Bayes' rule for any } T \text { and } s_{c}, \\
& \mu\left(\theta \mid s, s_{p}\right) \text { is obtained using Bayes' rule for any } s_{i} \text { and } s_{p} .
\end{aligned}
$$

Our focus will be on linear threshold equilibria. These are equilibria where speculators attack the currency if and only if their private signal $s_{i}$ is below a threshold $\hat{s}\left(s_{p}\right)$, which is a linear function of the common signal $s_{p}$. In addition, the central bank abandons the regime if and only if the aggregate size of the attack $T$ is above a threshold $\hat{T}\left(s_{c}\right)$, which is a function of its private signal $s_{c}$. The next proposition shows that there is a unique such equilibrium and characterizes it.

Proposition 1 There is a unique linear threshold equilibrium where the speculators' threshold strategy is:

$$
g\left(s, s_{p}\right)=\left\{\begin{array}{ll}
1 & \text { if } \quad s_{i} \leq \hat{s}\left(s_{p}\right) \\
0 & \text { if } \quad s_{i}>\hat{s}\left(s_{p}\right)
\end{array},\right.
$$

and the central bank's strategy is

$$
\delta\left(T, s_{c}\right)=\left\{\begin{array}{lll}
1 & \text { if } \quad T \leq \hat{T}\left(s_{c}\right) \\
0 & \text { if } \quad T>\hat{T}\left(s_{c}\right)
\end{array} .\right.
$$

Here,

$$
\hat{s}\left(s_{p}\right)=\hat{s}(0)-k s_{p}
$$

where $k>0$ is the unique real root of the cubic equation:

$$
-\frac{\tau_{c} \tau_{s}}{\tau_{p}} k^{3}+\left(\tau_{c}+\tau_{p}\right) k^{2}+2 \tau_{p} k+\tau_{p}=0
$$

and $\hat{s}(0)$ satisfies

$$
c=\Phi\left(\frac{-\left((1+k) \frac{\tau_{c}}{\tau} T+1\right) \frac{\tau_{s}}{\tau_{s}+\tau_{p}} \hat{s}(0)}{\sqrt{\left((1+k) \frac{\tau_{c}}{\tau_{T}}+1\right)^{2} \frac{1}{\tau_{s}+\tau_{p}}+(1+k)^{2} \frac{\tau_{c}}{\tau_{T}^{2}}}}\right),
$$


and

$$
\hat{T}\left(s_{c}\right)=\frac{1}{\sigma_{s}}\left[\hat{s}(0)+(1+k) \frac{\tau_{c}}{\tau_{T}} s_{c}\right],
$$

where

$$
\tau_{T}=\tau_{p}\left(1+\frac{1}{k}\right)^{2}
$$

is the precision of the attack as a signal of the fundamental.

\section{PROOF OF PROPOSITION 1:}

Suppose an agent attacks if and only if $s_{i}+k s_{p} \leq \hat{s}(0)$ where $k>0$. The size of the attack from speculators given $\theta$ and $s_{p}$ is $A\left(\theta, s_{p}\right)=\Phi\left(\frac{\hat{s}(0)-k s_{p}-\theta}{\sigma_{s}}\right)$. The central bank observes $T\left(\theta, s_{p}\right)=\Phi^{-1}(A)$, or equivalently, it observes

$$
T=\frac{\hat{s}(0)-k s_{p}-\theta}{\sigma_{s}}
$$

which can be rewritten as

$$
\frac{\hat{s}(0)-\sigma_{s} T}{1+k}=\theta+\frac{k \sigma_{p}}{1+k} \epsilon_{p} .
$$

Thus, the precision of the attack as a signal of the fundamental is

$$
\tau_{T}=\frac{\tau_{p}(1+k)^{2}}{k^{2}}
$$

and

$$
E\left[\theta \mid T, s_{c}\right]=\frac{\tau_{T}}{\tau_{T}+\tau_{c}}\left(\frac{\hat{s}(0)-\sigma_{s} T}{1+k}\right)+\frac{\tau_{c}}{\tau_{T}+\tau_{c}} s_{c} .
$$

This implies the status quo is abandoned if and only if

$$
T \geq \frac{\hat{s}(0)}{\sigma_{s}}+\frac{1+k}{\sigma_{s}} \frac{\tau_{c}}{\tau_{T}} s_{c}=\hat{T}\left(s_{c}\right)
$$

which is Equation (4). For a speculator, $\theta$ is distributed with mean $\frac{\tau_{s}}{\tau_{s}+\tau_{p}} s_{i}+\frac{\tau_{p}}{\tau_{s}+\tau_{p}} s_{p}$ and precision $\tau_{s}+\tau_{p}$. The posterior belief of the regime change for a speculator with signal $s_{i}$ 
and $s_{p}$ is expressed as follows:

$$
\begin{aligned}
& \operatorname{Pr}\left(T \geq \frac{\hat{s}(0)}{\sigma_{s}}+\frac{1+k}{\sigma_{s}} \frac{\tau_{c}}{\tau_{T}} s_{c} \mid s_{i}, s_{p}\right) \\
= & \operatorname{Pr}\left(\left((1+k) \frac{\tau_{c}}{\tau_{T}}+1\right) \theta+(1+k) \frac{\tau_{c}}{\tau_{T}} \sigma_{c} \epsilon_{c} \leq-k s_{p} \mid s_{i}, s_{p}\right) \\
= & \Phi\left(\frac{-\left((1+k) \frac{\tau_{c}}{\tau_{T}}+1\right) \frac{\tau_{s}}{\tau_{s}+\tau_{p}} s_{i}-\left(\left((1+k) \frac{\tau_{c}}{\tau_{T}}+1\right) \frac{\tau_{p}}{\tau_{s}+\tau_{p}}+k\right) s_{p}}{\sqrt{\left((1+k) \frac{\tau_{c}}{\tau_{T}}+1\right)^{2} \frac{1}{\tau_{s}+\tau_{p}}+\left((1+k) \frac{\tau_{c}}{\tau_{T}} \sigma_{c}\right)^{2}}}\right)
\end{aligned}
$$

In equilibrium the threshold strategy must satisfy the following equation for all $s_{p}$ :

$$
c=\Phi\left(\frac{-\left((1+k) \frac{\tau_{c}}{\tau_{T}}+1\right) \frac{\tau_{s}}{\tau_{s}+\tau_{p}} \hat{s}(0)-\left(\left((1+k) \frac{\tau_{c}}{\tau_{T}}+1\right)\left(\frac{\tau_{p}}{\tau_{s}+\tau_{p}}-\frac{\tau_{s}}{\tau_{s}+\tau_{p}} k\right)+k\right) s_{p}}{\sqrt{\left((1+k) \frac{\tau_{c}}{\tau_{T}}+1\right)^{2} \frac{1}{\tau_{s}+\tau_{p}}+\left((1+k) \frac{\tau_{c}}{\tau_{T}} \sigma_{c}\right)^{2}}}\right) .
$$

Thus, for a linear equilibrium to exist the coefficient of $s_{p}$ must be zero. In other words, $k$ must satisfy:

$$
-k \frac{\tau_{s}}{\tau_{s}+\tau_{p}}+\frac{\tau_{p}}{\tau_{s}+\tau_{p}}+\frac{k}{\left(1+(1+k) \frac{\tau_{c}}{\tau_{T}}\right)}=0 .
$$

Substituting for $\tau_{T}$ and rearranging the above equation we obtain:

$$
-\frac{\tau_{c} \tau_{s}}{\tau_{p}} k^{3}+\left(\tau_{c}+\tau_{p}\right) k^{2}+2 \tau_{p} k+\tau_{p}=0
$$

The above cubic equation has a single real root since the discriminant $\Delta$ given below is strictly positive:

$$
\Delta=\tau_{c}\left(4 \tau_{p}^{3}+8 \tau_{p}^{2} \tau_{c}+4 \tau_{p}^{2} \tau_{s}+4 \tau_{p} \tau_{c}^{2}+36 \tau_{p} \tau_{c} \tau_{s}+27 \tau_{c} \tau_{s}^{2}\right)>0 .
$$

To complete the construction of a linear equilibrium, we need to show that this root is strictly positive. Note that the left hand side of Equation (7) goes to $\infty$ as $k$ goes to $-\infty$ and $-\infty$ as $k$ goes to $\infty$. Moreover, it is positive at $k=0$. Since the equation has a single real root, it must cross zero at some $k>0$.

In equilibrium, the optimal strategy for a speculator who receives a signal $s_{i}$ is to attack if and only if $s_{i}$ falls below a threshold value, $\hat{s}\left(s_{p}\right)$, which is decreasing in the common noisy signal $s_{p}$. That is, when the common signal indicates a sound fundamental, speculators attack only if their private signals are very pessimistic. The weight $k$ that the speculator puts on the common signal is derived endogenously. For the central bank, the attack provides 
an additional signal about the fundamental. This signal has precision $\tau_{T}$ which is decreasing in the weight $k$ that speculators put on the common signal. The optimal strategy for the central bank is to abandon the exchange rate regime if and only if the observed signal of aggregate attack, $T$, is greater than or equal to the threshold, $\hat{T}\left(s_{c}\right)$, which is increasing in the central bank's private signal $s_{c}$.

\section{Informational Complementarities}

An important element of our equilibrium is $k$ - the weight that speculators put on the common signal $s_{p}$ in their decision whether to participate in a speculative attack. In general, speculators put a positive weight on $s_{p}$ because for them $s_{p}$ provides additional information about the realization of $\theta$, and thus on the probability that the central bank will abandon the exchange rate regime. It turns out, however, that in equilibrium speculators put too much weight on $s_{p}$. The reason is that speculators care about $s_{p}$, not only because of the information it provides about $\theta$, but also because of the information it provides about what other speculators are going to do - i.e., since speculators know that other speculators observe $s_{p}$ they know that $s_{p}$ affects other speculators' actions. Due to the presence of strategic complementarities, speculators want to do what they believe other speculators are going to do, and thus the weight they put on the common signal is higher than what it should be on the basis of pure informational content.

It is important to stress that, unlike in Morris and Shin (1998, 2002), strategic complementarities in our setting arise endogenously. They result from the fact that the central bank learns from the aggregate size of the attack about the fundamental $\theta$. Seeing a large attack, the central bank is more likely to believe that $\theta$ is low and to abandon the regime. Thus, when a speculator thinks that other speculators are more likely to attack, his incentive to attack also increases. We refer to these endogenous strategic complementarities as informational complementarities and distinguish them from the exogenous direct complementarities that are usually studied in the literature.

To demonstrate formally that informational complementarities increase the weight $k$ that speculators put on the common signal $s_{p}$, we now compare $k$ in our equilibrium (implicitly defined in Equation (3)) with a benchmark level $k_{B M}$ that would be obtained if the central bank did not use the size of the attack to infer information about the fundamental $\theta$. Intuitively, if the central bank did not learn from the size of the attack and used only its private 
signal, informational complementarities would disappear, since speculators would have no incentive to do what other speculators do. In such a model, the weight put by speculators on the common signal would be determined purely by the information that this signal provides about $\theta$. Thus, if informational complementarities have a bite, $k$ - defined in Equation (3) - should be greater than $k_{B M}$. The next proposition shows that this is indeed the case.

Proposition 2 The weight $k$ put by speculators on $s_{p}$ in the unique linear threshold equilibrium characterized by Proposition 1 is greater than the weight $k_{B M}$ that would be put on $s_{p}$ in a game where the central bank does not attempt to get information about $\theta$ from the size of the attack.

\section{PROOF OF PROPOSITION 2:}

We start by computing $k_{B M}$. When the central bank does not update its belief about $\theta$ based on the size of the attack, it will abandon the regime if and only if its private signal $s_{c}=\theta+\sigma_{c} \epsilon_{c}$ is negative. Then, based on the logic used in the proof of Proposition 1, speculators will attack the currency if and only if $\operatorname{Pr}\left(\theta+\sigma_{c} \epsilon_{c}<0 \mid s_{i}, s_{p}\right)>c$.

In a linear threshold equilibrium, speculators attack if and only if their private signal $s_{i}$ is below the threshold $\hat{s}_{B M}\left(s_{p}\right)=\hat{s}_{B M}(0)-k_{B M} s_{p}$. Thus, the speculator who observes the threshold signal has to be indifferent between attacking and not attacking:

$$
\operatorname{Pr}\left(\theta+\sigma_{c} \epsilon_{c}<0 \mid \hat{s}_{B M}\left(s_{p}\right), s_{p}\right)=c .
$$

Since $\theta$ and $\epsilon_{c}$ are independently normally distributed, this implies that:

$$
\Phi\left(\frac{-\frac{\tau_{s}}{\tau_{s}+\tau_{p}} \hat{s}_{B M}\left(s_{p}\right)-\frac{\tau_{p}}{\tau_{s}+\tau_{p}} s_{p}}{\sqrt{\frac{1}{\tau_{s}+\tau_{p}}+\sigma_{c}^{2}}}\right)=c .
$$

Plugging in $\hat{s}_{B M}\left(s_{p}\right)=\hat{s}_{B M}(0)-k_{B M} s_{p}$, we get:

$$
\Phi\left(\frac{-\frac{\tau_{s}}{\tau_{s}+\tau_{p}} \hat{s}_{B M}(0)-\left(\frac{\tau_{p}}{\tau_{s}+\tau_{p}}-\frac{\tau_{s}}{\tau_{s}+\tau_{p}} k_{B M}\right) s_{p}}{\sqrt{\frac{1}{\tau_{s}+\tau_{p}}+\sigma_{c}^{2}}}\right)=c .
$$

Since this equation has to hold for every $s_{p}$, we get:

$$
k_{B M}=\frac{\tau_{p}}{\tau_{s}} .
$$

Now, we show that $k$ (defined in Equation (3)) is greater than $k_{B M}$. To do this, we need to 
evaluate the left side of Equation (3) at $k=k_{B M}=\frac{\tau_{p}}{\tau_{s}}$. This yields:

$$
\begin{aligned}
& -\frac{\tau_{c} \tau_{p}^{2}}{\tau_{s}^{2}}+\left(\tau_{c}+\tau_{p}\right) \frac{\tau_{p}^{2}}{\tau_{s}^{2}}+2 \frac{\tau_{p}^{2}}{\tau_{s}}+\tau_{p} \\
= & \frac{\tau_{p}}{\tau_{s}^{2}}\left(\tau_{p}^{2}+2 \tau_{p} \tau_{s}+\tau_{s}^{2}\right)>0 .
\end{aligned}
$$

Hence, $k>k_{B M}$.

In sum, despite the fact that our model does not assume direct strategic complementarities among speculators, we get the result that speculators pay too much attention to the common signal and too little attention to their private signals. The fact that the central bank learns from speculators' actions creates endogenous informational complementarities that generate this result. In the next section, we will see that these informational complementarities have an important effect: They reduce the efficiency of the central bank's decision on whether or not to abandon the exchange rate regime.

Before turning to the next section, it is important to understand the subtlety of the notion of informational complementarities in our paper and the difference from the notion of direct complementarities in Morris and Shin $(1998,2002)$ and the rest of the literature. Under direct complementarities, the incentive of a speculator to attack the regime changes with any change in other speculators' strategies. This is because the actions of other speculators have a direct effect on the viability of the regime, and thus change the profitability of attacking it. On the other hand, in our framework a change in speculators' strategies affects the incentives of other speculators only if it affects the central bank's belief about $\theta$. A key insight is then that strategic complementarities arise only if there is a piece of information that is shared by speculators, but not known to the central bank. Speculators can then coordinate on changing their behavior based on the realization of this piece of information and essentially act as if they 'manipulate' the central bank's belief. In our model, they coordinate on attacking more (less) aggressively when the common signal they observe is low (high). Since the central bank does not observe the common signal, it does not know if a large attack is due to coordination or information, and so it can be "fooled" to take the wrong action.

The assumption that the central bank does not observe a signal that is commonly observed by all speculators may seem a bit extreme. It may be justified by the fact that in these settings speculators usually do not operate in the domestic market, and thus may have information that is not available to the central bank. More importantly, we wish to stress that this assumption is not crucial to our model, but it greatly simplifies the exposition. 
The essential element of the model is that the speculators are able to coordinate their actions through some commonality of their information and the central bank does not fully observe this commonality. For our results to go through, this commonality does not need to be extreme and it can be achieved with alternative information structures. For example, our results can be preserved in a symmetric information environment where both the central bank and the speculators observe the common signal with some given probability less than one. In this case, since there are a continuum of speculators, a fixed proportion of the speculators always observe the common signal. Moreover, the speculators know that with a positive probability the central bank has missed this signal; hence, there is still room for the coordination motive to play a role, and the basic insights of our analysis continue to hold. This setup is attractive because the central bank and the speculators are symmetrically informed. Alternatively, our results will go through in an information environment where the common signal is observed with some idiosyncratic noise by all agents including speculators and the central bank. In this framework, speculators can still coordinate their actions by putting excess weight on the noisy common signal, and the central bank cannot filter it out completely since it is not fully informed about the common component of the signals. Finally, in Appendix B, we show that a very similar result holds even when the speculators receive a common signal which is not fundamental related. ${ }^{9}$ That assumption is empirically plausible since speculators may share some common information regarding random shocks to the institutional environment or to the workings of the currency market, which are not known to the central bank (for example, traders may know other traders personally, and thus expect changes in their appetite for risk). ${ }^{10}$

We close this section by analyzing the determinants of $k$ in equilibrium.

Proposition 3 The equilibrium value of $k$ decreases in $\tau_{c}$ and $\tau_{s}$, and increases in $\tau_{p}$.

\section{PROOF OF PROPOSITION 3}

\footnotetext{
${ }^{9}$ In that case, we assume that the central bank observes the size of the attack with noise and the speculators are able to observe the noise component of the size of the attack.

${ }^{10}$ Related to this, one may ask whether the central bank can construct a mechanism to reveal the common signal by directly contacting speculators. While addressing this point formally is beyond the scope of our paper, we wish to point to the difficulty of implementing such mechanism in the real world. A key feature of financial markets is the anonymity of most speculators. This implies that it may be difficult for the central bank to identify the speculators who are active in the market at a particular point in time.
} 
By taking the total derivative of Equation (7) with respect to $\tau_{c}$ we obtain:

$$
\frac{\partial k}{\partial \tau_{c}}=\frac{\frac{\tau_{s}}{\tau_{p}} k^{3}-k^{2}}{-3 \frac{\tau_{c} \tau_{s}}{\tau_{p}} k^{2}+2\left(\tau_{c}+\tau_{p}\right) k+2 \tau_{p}}
$$

To see the sign of the denominator of Equation (8) in equilibrium, we use the equilibrium condition given by Equation (7) to obtain:

$$
-3 \frac{\tau_{c} \tau_{s}}{\tau_{p}} k^{2}+2\left(\tau_{c}+\tau_{p}\right) k+2 \tau_{p}=\frac{-\left(\tau_{c}+\tau_{P}\right) k^{2}-4 \tau_{P} k-3 \tau_{P}}{k}<0 .
$$

Similarly, to see the sign of the numerator of Equation (8) in equilibrium, we once more use Equation (7) to obtain:

$$
\frac{\tau_{s}}{\tau_{p}} k^{3}-k^{2}=\frac{\tau_{p} k^{2}+2 \tau_{p} k+\tau_{p}}{\tau_{c}}>0 .
$$

Thus, $\frac{\partial k}{\partial \tau_{c}}<0$. Similarly,

$$
\begin{aligned}
\frac{\partial k}{\partial \tau_{p}} & =\frac{\frac{\tau_{c} \tau_{s}}{\tau_{p}^{2}} k^{3}+k^{2}+2 k+1}{3 \frac{\tau_{c} \tau_{s}}{\tau_{p}} k^{2}-2\left(\tau_{c}+\tau_{p}\right) k-2 \tau_{p}}>0, \\
\frac{\partial k}{\partial \tau_{s}} & =\frac{\frac{\tau_{c}}{\tau_{p}} k^{3}}{-3 \frac{\tau_{c} \tau_{s}}{\tau_{p}} k^{2}+2\left(\tau_{c}+\tau_{p}\right) k+2 \tau_{p}}<0 .
\end{aligned}
$$

In other words, if the central bank's or the speculators' private information is less precise, or the common signal is more precise, speculators coordinate better in equilibrium so that the weight they put on the common signal increases. Intuitively, if the central bank holds a precise signal, it relies less on the information revealed in the aggregate actions of the speculators. In equilibrium, this forces the speculators to reduce the weight they put on the common signal and to reveal more information to the central bank. If each speculator holds a very sharp private signal about the fundamental, each bases the decision to attack mostly on the private signal rather than the noisy common signal, and hence there is less incentive to coordinate. Finally, the incentive to coordinate is largest when the common signal is very precise. In this case, the speculators put a larger weight on the common signal and the central bank cannot ignore the information revealed in the aggregate attack.

\section{The Efficiency of the Central Bank's Decision}

We now examine the relationship between informational complementarities and the efficiency of the central bank's policy decision. We measure inefficiency using the probability of aban- 
doning (maintaining) the status quo given that $\theta>0(\theta<0)$ and before any signal is observed. If $\theta>0(\theta<0)$, abandoning (maintaining) the status quo is a policy error. Thus, our measure captures the probability of making policy mistakes. The following proposition characterizes the probability of making a policy mistake and studies its properties.

Proposition 4 The ex ante probability of abandoning the status quo for a given $\theta$ is

$$
\Phi\left(-\sqrt{\tau_{c}+\tau_{T}} \theta\right) .
$$

Hence, when $\theta>0$, the probability of making a policy mistake is $\Phi\left(-\sqrt{\tau_{c}+\tau_{T}} \theta\right)$, while when $\theta<0$, it is $1-\Phi\left(-\sqrt{\tau_{c}+\tau_{T}} \theta\right)$. Then,

1) The probability of making a policy mistake is positive as long as $\tau_{c}$ and $\tau_{p}$ do not approach $\infty$ and $k$ does not approach 0 .

2) The probability of making a policy mistake increases in $k$.

\section{PROOF OF PROPOSITION 4:}

The ex ante probability of abandoning the status quo given $\theta$ is:

$$
\operatorname{Pr}\left(T \geq \frac{\hat{s}(0)}{\sigma_{s}}+\frac{1+k}{\sigma_{s}} \frac{\tau_{c}}{\tau_{T}} s_{c} \mid \theta\right)=\operatorname{Pr}\left(\epsilon_{c}+\frac{1}{\sigma_{c}} \frac{\sqrt{\tau_{T}}}{\tau_{c}} \epsilon_{p} \leq-\frac{1}{\sigma_{c}}\left(1+\frac{\tau_{T}}{\tau_{c}}\right) \theta \mid \theta\right)
$$

where the equality follows by plugging in for $T, s_{p}, s_{c}$ and rearranging Equation (6). The term $\epsilon_{c}+\frac{1}{\sigma_{c}} \frac{\sqrt{\tau_{T}}}{\tau_{c}} \epsilon_{p}$ is the weighted sum of two independent normal random variables, so this term itself is normal with mean 0 and variance $1+\frac{\tau_{T}}{\tau_{c}}$. Thus the ex-ante probability of abandoning the status quo for a given $\theta$ is given by:

$$
\operatorname{Pr}\left(\epsilon_{c}+\frac{1}{\sigma_{c}} \frac{\sqrt{\tau_{T}}}{\tau_{c}} \epsilon_{p} \leq-\frac{1}{\sigma_{c}}\left(1+\frac{\tau_{T}}{\tau_{c}}\right) \theta \mid \theta\right)=\Phi\left(-\sqrt{\tau_{c}+\tau_{T}} \theta\right) .
$$

The rest of the proposition follows directly from the definition of $\tau_{T}$ in Equation (5) where $\tau_{T}=\tau_{p}(1+1 / k)^{2}$

Intuitively, the probability of making a policy mistake is a function of the precision of the two pieces of information that the central bank has: the precision of the information conveyed by the size of the attack $\tau_{T}$ and the precision of the central bank's private information $\tau_{c}$. As long as these two precisions do not approach $\infty$, the central bank makes mistakes in its policy decision with a positive probability. Inspecting the expression for the precision of the size of the attack in Equation (5), we can see that the attack is not fully revealing unless the precision of the common signal $\tau_{p}$ approaches infinity, or the speculators put no weight on the common signal in their attack decision, i.e., unless $k$ approaches 0. Moreover, the 
probability of making a policy mistake is increasing in $k$. This result demonstrates the real effect of informational complementarities. In Proposition 2, we showed that informational complementarities contribute to an increase in the weight $k$ that speculators put on the common signal. Now we say that the inefficiency in the central bank's decision increases in $k$. Taken together, these results imply that informational complementarities reduce the efficiency of the central bank's policy decision. ${ }^{11}$

To see the intuition behind this result, it is useful to consider what would happen in a model where the common signal played no role. It turns out that such a model is in fact a limit case of our model which is obtained by letting $\tau_{p}$ approach 0 . As the next Proposition shows, in this case, the common signal becomes too noisy and the speculators can no longer coordinate on it, i.e., $k \rightarrow 0$. The result is that the attack becomes fully revealing of the fundamental and the central bank does not make policy mistakes.

Proposition 5 In the limit as $\tau_{p}$ approaches $0, \hat{s}\left(s_{p}\right)=\hat{s}(0)=\sigma_{s} \Phi^{-1}(1-c), \hat{T}\left(s_{c}\right)=$ $\Phi^{-1}(1-c)$, and the attack becomes fully revealing of $\theta$.

\section{PROOF OF PROPOSITION 5:}

We start with the following lemma:

Lemma 1 (i) $\lim _{\tau_{p} \rightarrow 0} k\left(\tau_{p}\right)=0$, (ii) $\lim _{\tau_{p} \rightarrow 0} k\left(\tau_{p}\right) / \tau_{p}=\infty$, and (iii) $\lim _{\tau_{p} \rightarrow 0} k\left(\tau_{p}\right)^{2} / \tau_{p}=0$.

PROOF OF LEMMA 1 To see that $k\left(\tau_{p}\right)$ approaches zero as $\tau_{p}$ approaches zero, first recall that $\partial k / \partial \tau_{p}$ is positive. Thus as $\tau_{p}$ approaches zero, $k\left(\tau_{p}\right)$ has a limit that is less than infinity. Suppose that this limit is strictly positive. Then it is easy to see that

$$
-\frac{\tau_{c} \tau_{s}}{\tau_{p}} k^{3}+\left(\tau_{c}+\tau_{p}\right) k^{2}+2 \tau_{p} k+\tau_{p}<0
$$

for $\tau_{p}$ small enough which is a contradiction, establishing that $\lim _{\tau_{p} \rightarrow 0} k\left(\tau_{p}\right)=0$.

By letting $z=\frac{k}{\tau_{p}}$, Equation (3) can be rewritten as

$$
-\tau_{c} \tau_{s} z^{3}+\left(\tau_{c}+\tau_{p}\right) z^{2}+2 z+\frac{1}{\tau_{p}}=0
$$

\footnotetext{
${ }^{11}$ It is useful to note that informational complementarities are not the only source of inefficiency in the model. As long as the precision of the common signal is bounded from zero, speculators will put a positive weight on it (see the definition of $k_{B M}$ above), and this will generate policy mistakes. Yet, the presence of complementarities amplifies the weight they put on the common signal, and thus increases the inefficiency.
} 
From the above equation we see that as $\tau_{p}$ approaches zero, $z=\frac{k}{\tau_{p}}$ approaches $\infty$. Moreover, from Equation (3),

$$
\left(\frac{k^{2}}{\tau_{p}}\right)=\frac{(2 k+1)}{\tau_{c} \tau_{s} \frac{k}{\tau_{p}}-\left(\tau_{c}+\tau_{p}\right)}
$$

and the right side approaches zero as $\tau_{p}$ approaches zero, establishing $\lim _{\tau_{p} \rightarrow 0} k\left(\tau_{p}\right)^{2} / \tau_{p}=0$.

Using Lemma 1, we see that as $\tau_{p}$ approaches zero,

$$
c=\Phi\left(\frac{-\left(\frac{\tau_{c}}{(1+k)} \frac{k^{2}}{\tau_{p}}+1\right) \frac{\tau_{s}}{\tau_{s}+\tau_{p}} \hat{s}(0)}{\sqrt{\left(\frac{\tau_{c}}{(1+k)} \frac{k^{2}}{\tau_{p}}+1\right)^{2} \frac{1}{\tau_{s}+\tau_{p}}+\tau_{c} \frac{\hat{k}^{2}}{\tau_{p}}}}\right) \rightarrow 1-\Phi\left(\sqrt{\tau_{s}} \hat{s}(0)\right)
$$

and

$$
\hat{T}\left(s_{c}\right)=\frac{1}{\sigma_{s}}\left[\hat{s}(0)+\frac{k^{2} \tau_{c}}{\tau_{p}(1+k)} s_{c}\right] \rightarrow \frac{1}{\sigma_{s}} \hat{s}(0)=\Phi^{-1}(1-c) .
$$

To see that the limiting equilibrium is fully revealing, note that a speculator attacks if and only if $s \leq \hat{s}(0)$. The size of the attack from speculators is $A(\theta)=\Phi\left(\frac{\hat{s}(0)-\theta}{\sigma_{s}}\right)$, and the central bank observes $T(\theta)=\Phi^{-1}(A)$ or $T=(\hat{s}(0)-\theta) / \sigma_{s}$. Hence, in equilibrium, the central bank infers $\theta$ perfectly which is given by:

$$
\theta=\hat{s}-\sigma_{s} T
$$

Inspecting Proposition 4 and Proposition 5 together, we can see that if speculators put weight only on their private signals, then their aggregate action enables the central bank to learn $\theta$ perfectly. This is because the idiosyncratic noise terms in the speculators' private signals cancel out with each other and end up having no effect on the aggregate size of the attack. On the other hand, once speculators put weight on the common signal, the noise in the common signal has an effect on the aggregate size of the attack. This prevents the central bank from perfectly learning $\theta$. Intuitively, the problem becomes more severe once the weight on the common signal becomes larger, which happens as a result of informational complementarities. ${ }^{12}$

Taken together, the two propositions also generate a puzzling result. Even though speculators are better informed about the fundamental when they observe a common signal in

\footnotetext{
${ }^{12}$ In Appendix C, we study the other limit where the common signal becomes infinitely precise and hence the speculators commonly know the fundamental. We show that in this limit the linear equilibrium disappears, and there are multiple equilibria. In these equilibria, speculators coordinate their actions perfectly by either all of them or none of them attacking for a given value of the fundamental.
} 
addition to their private signals, the central bank, paradoxically, becomes less informed when this signal is introduced. This suggests that adding a source of information reduces the efficiency of the policy decision taken by the central bank. We now explore this aspect of the model more fully by analyzing the effect that the precision of the various signals in our model have on the efficiency of the policy decision. The following proposition provides the main result.

Proposition 6 Conditional on $\theta$, the ex-ante probability of making a policy mistake decreases in $\tau_{c}$ and $\tau_{s}$, increases in $\tau_{p}$ if $0<\tau_{p}<\tau_{c} \frac{\sqrt{1+16 \frac{\tau_{s}}{\tau_{c}}}-1}{8}$ and decreases in $\tau_{p}$ if $\tau_{p}>$ $\tau_{c} \frac{\sqrt{1+16 \frac{\tau_{s}}{\tau_{c}}}-1}{8}$.

\section{PROOF OF PROPOSITION 6: See Appendix A.}

The results regarding $\tau_{s}$ and $\tau_{c}$ are straightforward: Improving the precision of the speculators' private signals or of the central bank's private signal generates an increase in the efficiency of the central bank's policy decision. But the effect of increasing the precision $\tau_{p}$ of the common signal can go in both directions. On the one hand, increasing the precision of the common signal implies that speculators have access to more precise information, which can be revealed to the central bank via the trading process. On the other hand, increasing the precision of the common signal implies that speculators will rely more on the common signal, partly because of the presence of informational complementarities - that is, the ability of speculators to coordinate on the common signal and convey a misleading message to the central bank improves. Our result shows that the first effect dominates when $\tau_{p}$ is above a certain cutoff and the second effect dominates when $\tau_{p}$ is below the cutoff. Thus, an increase in the precision of the common signal enhances efficiency if and only if this precision is above a certain cutoff. This result is related to the one in Morris and Shin (2002). The big difference is that in our model there are no assumed strategic complementarities. Rather, strategic complementarities arise endogenously as a result of the learning process.

We conclude this section by pointing to another interesting aspect of our model, which stands in stark difference to existing models of currency attacks. This is summarized in the next corollary.

Corollary 1 The level of the opportunity cost, c (as well as the wealth level of speculators) affects the size of the attack but does not affect the information content of the attack and, hence, does not affect the probability of devaluation occurring for a given $\theta$. 
Corollary 1 offers an explanation to an empirically puzzling fact that some speculative attacks are defended by the central banks while some, that are even stronger, are not. According to the findings in our model, if the opportunity cost is low or speculators have more wealth, attacks will be larger, but their information content remains unchanged. Therefore, the status quo will be defended when large attacks result from the fact that there is more hot money or that it is less costly to short sell. This result offers some testable implications regarding governments' policy decisions and the information content of currency trading, which distinguish this paper from the existing literature. Market microstructure papers, for example, typically use measures of informativeness constructed from frequencies and directions of trades, which are not perfectly correlated with trading volumes or proxies for size of speculative attack (Easley and O'Hara (1987); O'Hara (1995)). Bid-ask spreads and dispersion of analysts' forecasts also can be used as a measure of informativeness in currency trades. One can test whether these market microstructure measures are related to the government's decision to defend its exchange rate regime or not, or the length of the defense if it chooses not to abandon.

\section{$6 \quad$ Policy Implications}

We now analyze the effect of two policy tools on the efficiency of the central bank's decision in our model.

\subsection{Commitment}

Knowing that the central bank uses the information in the size of the attack, speculators put a too large weight on the common signal. This, in turn, reduces the informativeness of the size of the attack and harms the efficiency of the central bank's policy decision. The first policy tool that we explore is commitment by the central bank to reduce the weight it puts on the information in the size of the attack below what is ex-post optimal. There are two effects to consider in evaluating this policy tool. First, there is obviously a cost in deviating from the ex-post optimal decision. When doing that, the central bank does not fully use the information available to it, and this entails an efficiency loss. Second, by committing to put a low weight on the size of the attack, the central bank affects the ex-ante trading motives of speculators. In particular, under such policy, speculators will find it less valuable to coordinate on the common signal, and this will improve the quality of the information 
conveyed by the attack. The next proposition analyzes the overall desirability of such a commitment policy.

Proposition 7 Suppose that speculators follow linear strategies, then the central bank can always increase the ex-ante efficiency of its policy decision by committing to put a slightly lower (higher) weight on the information in the attack (on its private signal) than is ex-post optimal.

PROOF OF PROPOSITION 7: See Appendix A.

The proposition says that it is always optimal for the central bank to commit to a slight deviation from the ex-post optimal weights, and in particular to increase (decrease) the weight given to its private signal (to the information in the size of the attack). The reason is that the cost of deviating from ex-post efficiency approaches 0 when it is evaluated at a point that is close to ex-post efficiency. On the other hand, the benefit from the increased informativeness of the attack is always strictly bounded from 0 . As a result, slight deviations from ex-post efficiency are always desirable. Of course, large deviations are not desirable. For example, it is never optimal for the central bank to completely ignore the attack as a signal for the fundamentals. This can be seen by comparing the probability of devaluation for a given $\theta$ when the central bank ignores the attack which is given by $\Phi\left(-\sqrt{\tau_{c}} \theta\right)$ and the probability of devaluation when the central bank learns from the attack which is given by $\Phi\left(-\sqrt{\tau_{c}+\tau_{T}} \theta\right)$. The latter probability is smaller when $\theta>0$ and larger when $\theta<0$. Finally, note that the feasibility of the policy tool analyzed here depends critically on the central bank's commitment power. This is because this policy tool entails time inconsistency: $E x$ post, once the attack is realized, the central bank would always be tempted to pay more attention to the attack.

\subsection{Transparency}

Next, we discuss the issue of central bank transparency, in other words, whether and how clearly the central bank should communicate its information to the public. The issue of central bank transparency is receiving a lot of attention in research and policy circles. The positive aspects of transparency are often emphasized. Recently, Morris and Shin (2005) demonstrate a cost associated with transparency. Building on the insight in Morris and Shin (2002), they show that in the presence of direct complementarities among market participants, transparency can be bad because it provides a public signal, and thus reduces 
the extent to which speculators use their private information. This, in turn, reduces the ability of the central bank to infer new information from the market.

We analyze the effect of transparency in our framework, where there are no direct complementarities among speculators, but rather speculators care about each other's strategies because they know that their collective action reveals information that affects the central bank's policy decision. We discover a new negative effect of transparency. When the central bank becomes more transparent, it reveals information about the course of action that it is likely to take in the future. Knowing this, the speculators can better coordinate on conveying a misleading signal. To see this, consider the extreme case where the central bank perfectly reveals its information, and suppose that speculators know that solely on the basis of this information, the central bank would devalue. In this case, it is an equilibrium for all the speculators to attack. Since the attack reveals no information the central bank will indeed devalue, and completely miss the opportunity to learn from the market to shape its policy decision. The next proposition analyzes the more interesting case where the central bank releases its information with some noise. It shows that as long as this information is commonly observed by the speculators, the central bank's decisions become less efficient.

Proposition 8 Suppose central bank releases a public signal $s_{a}=s_{c}+\sigma_{a} \epsilon_{a}$ where $\epsilon_{a} \sim$ $N(0,1)$. The weight $k_{t r}$ put by speculators on $s_{p}$ in the unique linear threshold equilibrium is bigger than that characterized by Proposition 1.

\section{PROOF OF PROPOSITION 8: See Appendix A.}

The proposition indicates that the central bank may inadvertently strengthen the coordination incentive by releasing more information that becomes common to the speculators. In fact, the more precise such information is, the stronger the coordination among speculators (i.e., the higher $k$ ). As discussed above, this result is due to the fact that speculators can coordinate more efficiently when they have common information about the central bank's signal, since this information is very revealing about the central bank's action.

Finally, we would like to stress that the negative effect of transparency on efficiency is due to the fact that the central bank releases information which becomes common knowledge to all speculators. One could imagine a different form of transparency, by which the central bank releases information that is interpreted differently by different speculators. In such a case, transparency increases the precision of speculators' private signals and the above 
conclusion is overturned. Thus, our model has strong implications regarding the optimal form of transparency.

The next proposition analyzes the second case and shows that if the additional signal provided by the central bank has a noise term that is specific to each speculator, then transparency improves the precision of private information for each speculator and weakens the incentive for them to coordinate.

Proposition 9 Suppose central bank releases a public signal $s_{a}=s_{c}+\sigma_{a} \epsilon_{a i}$ where $\epsilon_{a i} \sim$ $N(0,1)$ and drawn independently across speculators. The weight that the speculators put on $s_{p}$ in the unique linear threshold equilibrium is smaller than the one characterized in Proposition 1.

\section{PROOF OF PROPOSITION 9:}

The variance of the speculator's private information about $\theta$ (that is, two combined private signals $)$ is $1 / \tau_{s a}=1 /\left(\tau_{s}+\tau_{a}\right)$, smaller than $1 / \tau_{s}$, the variance of their private information as characterized in Proposition 1. The result then follows from Proposition 3

\section{Conclusion}

We analyze a model where the information revealed in the course of a speculative attack is used by the central bank in its policy decisions. On the one hand, this information enhances the efficiency of the central bank's policy decisions. On the other hand, the fact that the central bank uses the information gives rise to endogenous strategic complementarities which we call informational complementarities - due to which speculators wish to coordinate on similar trading actions even if they conflict with their private information. These coordination motives reduce the informational content of the speculators' collective action and the efficiency of the central bank policy decisions. We analyze the tradeoff between information and coordination in speculative attacks, and derive comparative statics regarding the behavior of speculators and on the efficiency of the central bank's policy decisions. We also analyze the effect of different policy measures that the central bank may adopt to improve the efficiency of its decisions.

Our paper contributes to three different literatures. First, we introduce an important channel to the literature on currency attacks - namely, the learning by the central bank from market activities. We show the positive and negative aspects of such learning and 
study the nature of speculative attacks that it generates. Second, we provide a new angle to the literature on the feedback effect from financial markets to the real economy. We show that the fact that a decision maker learns from the trading process and takes an action that affects the value of traded securities gives rise to coordination problems among market participants, which result in destabilizing trading and reduced efficiency. Thus, the analysis here can be used to study other settings where decision makers learn from the aggregate trade in financial markets. Examples include learning by firm managers and providers of capital when deciding whether to go ahead with an investment project. Finally, by endogenizing strategic complementarities to be a result of information aggregation and learning, we provide micro foundations for the presence of strategic complementarities, and thus contribute to the literature on coordination games (and in particular, global games) that considered complementarities to be exogenous. We show the differences between the endogenous informational complementarities in our framework and the exogenous direct complementarities studied previously in the literature. 


\section{Appendix A}

\section{Proofs}

\section{PROOF OF PROPOSITION 6:}

Inspecting the expressions for the probability of a policy mistake in Proposition 4, we see that in order to prove the current proposition, we need to show that $\tau_{T}$ increases in $\tau_{c}$ and $\tau_{s}$, decreases in $\tau_{p}$ if $0<\tau_{p}<\tau_{c} \frac{\sqrt{1+16 \frac{\tau_{s}}{\tau_{c}}}-1}{8}$ and increases in $\tau_{p}$ if $\tau_{p}>\tau_{c} \frac{\sqrt{1+16 \frac{\tau_{s}}{\tau_{c}}}-1}{8}$.

From Equation (5), note that

$$
\frac{\partial \tau_{T}}{\partial \tau_{s}}=-2 \tau_{p} \frac{(1+k)}{k^{2}} \frac{1}{k} \frac{\partial k}{\partial \tau_{s}}>0 \text { and } \frac{\partial \tau_{T}}{\partial \tau_{c}}=-2 \tau_{p} \frac{(1+k)}{k^{2}} \frac{1}{k} \frac{\partial k}{\partial \tau_{c}}>0 .
$$

Next note,

$$
\frac{\partial \tau_{T}}{\partial \tau_{p}}=\frac{(1+k)}{k^{2}}\left[(1+k)-2 \tau_{p} \frac{1}{k} \frac{\partial k}{\partial \tau_{p}}\right]
$$

and from Equation (3),

$$
\frac{\partial k}{\partial \tau_{p}}=\frac{\frac{\tau_{c} \tau_{s}}{\tau_{p}^{2}} k^{3}+k^{2}+2 k+1}{3 \frac{\tau_{c} \tau_{s}}{\tau_{p}} k^{2}-2\left(\tau_{c}+\tau_{p}\right) k-2 \tau_{p}} .
$$

Substituting and rearranging we obtain:

$$
\frac{\partial \tau_{T}}{\partial \tau_{p}}=\frac{(1+k)}{k^{2}}\left[\frac{3 \frac{\tau_{c} \tau_{s}}{\tau_{p}} k^{4}+\frac{\tau_{c} \tau_{s}}{\tau_{p}} k^{3}-2\left(\tau_{c}+\tau_{p}\right) k^{3}-2\left(\tau_{c}+\tau_{p}\right) k^{2}-4 \tau_{p} k^{2}-6 \tau_{p} k-2 \tau_{p}}{3 \frac{\tau_{c} \tau_{s}}{\tau_{p}} k^{3}-2\left(\tau_{c}+\tau_{p}\right) k^{2}-2 \tau_{p} k}\right] .
$$

We know that $k>0$. Moreover, we know from the proof of Proposition 3 that $3 \frac{\tau_{c} \tau_{s}}{\tau_{p}} k^{3}-$ $2\left(\tau_{c}+\tau_{p}\right) k^{2}-2 \tau_{p} k>0$. So the sign of $\frac{\partial \tau_{T}}{\partial \tau_{p}}$ is the same as the sign of

$$
3 \frac{\tau_{c} \tau_{s}}{\tau_{p}} k^{4}+\frac{\tau_{c} \tau_{s}}{\tau_{p}} k^{3}-2\left(\tau_{c}+\tau_{p}\right) k^{3}-2\left(\tau_{c}+\tau_{p}\right) k^{2}-4 \tau_{p} k^{2}-6 \tau_{p} k-2 \tau_{p} .
$$

We can rewrite this expression as:

$$
\begin{aligned}
& 3 \frac{\tau_{c} \tau_{s}}{\tau_{p}} k^{4}-3\left(\tau_{c}+\tau_{p}\right) k^{3}-6 \tau_{p} k^{2}-3 \tau_{p} k \\
& +\frac{\tau_{c} \tau_{s}}{\tau_{p}} k^{3}+\left(\tau_{c}+\tau_{p}\right) k^{3}+2 \tau_{p} k^{2}-2\left(\tau_{c}+\tau_{p}\right) k^{2}-3 \tau_{p} k-2 \tau_{p} .
\end{aligned}
$$

The first line of this expression is zero from Equation (3). We can rewrite the second line as:

$$
\begin{aligned}
& \frac{\tau_{c} \tau_{s}}{\tau_{p}} k^{3}-\left(\tau_{c}+\tau_{p}\right) k^{2}-2 \tau_{p} k-\tau_{p} \\
& +\left(\tau_{c}+\tau_{p}\right) k^{3}+2 \tau_{p} k^{2}-\left(\tau_{c}+\tau_{p}\right) k^{2}-\tau_{p} k-\tau_{p} .
\end{aligned}
$$

Once again the first line is zero by Equation (3). The second line can be rewritten as:

$$
(k-1)\left(\tau_{c} k^{2}+\tau_{p}(k+1)^{2}\right) .
$$


Thus the sign of $\frac{\partial \tau_{T}}{\partial \tau_{p}}$ is the same as the sign of $(k-1)$. To determine the sign of $(k-1)$, we evaluate the left side of Equation (3) at $k=1$ which is

$$
\frac{4 \tau_{p}^{2}+\tau_{c} \tau_{p}-\tau_{c} \tau_{s}}{\tau_{p}} .
$$

If the value of the above expression is positive then $k>1$, and if it is negative then $k<1$. (This is because the cubic function on left side of Equation (3) crosses zero only once and from above.) It is easy to see that the above expression is negative if

$$
0<\tau_{p}<\tau_{c} \frac{\sqrt{1+16 \frac{\tau_{s}}{\tau_{c}}}-1}{8}
$$

and positive if

$$
\tau_{p}>\tau_{c} \frac{\sqrt{1+16 \frac{\tau_{s}}{\tau_{c}}}-1}{8}
$$

Thus the sign of $\frac{\partial \tau_{T}}{\partial \tau_{p}}$ is negative if $0<\tau_{p}<\tau_{c} \frac{\sqrt{1+16 \frac{\tau_{s}}{\tau_{c}}}-1}{8}$ and positive if $\tau_{p}>\tau_{c} \frac{\sqrt{1+16 \frac{\tau_{s}}{\tau_{c}}}-1}{8}$

\section{PROOF OF PROPOSITION 7:}

Suppose that speculators follow linear strategies, that is, a speculator attacks if and only if his signal $s_{i}$ is below $s(0)-k s_{p}$. As before, the central bank observes:

$$
\frac{s(0)-\sigma_{s} T}{1+k}=\theta+\frac{k \sigma_{p}}{1+k} \epsilon_{p} .
$$

Suppose that the central bank abandons the status quo if and only if

$$
\left(\frac{\tau_{T}-\beta}{\tau_{T}+\tau_{c}}\right)\left(\frac{\hat{s}(0)-\sigma_{s} T}{1+k}\right)+\left(\frac{\tau_{c}+\beta}{\tau_{T}+\tau_{c}}\right) s_{c} \leq 0
$$

or

$$
T \geq \frac{1}{\sigma_{s}} s(0)+\frac{(1+k)}{\sigma_{s}} \frac{\left(\tau_{c}+\beta\right)}{\left(\tau_{T}-\beta\right)} s_{c}
$$

for $\tau_{T}>\beta>0$ where $\tau_{T}=\tau_{p} \frac{(1+k)^{2}}{k^{2}}$. Here, $\beta$ is the deviation from ex-post optimal weights. It measures the increase (decrease) in the weight given to the central bank's private signal (to the information in the attack) relative to the ex-post optimal level. The posterior belief 
of the regime change for a speculator with signal $s$ and $s_{p}$ is now expressed as follows:

$$
\begin{aligned}
& \operatorname{Pr}\left(T \geq \frac{1}{\sigma_{s}} s(0)+\frac{(1+k)}{\sigma_{s}} \frac{\left(\tau_{c}+\beta\right)}{\left(\tau_{T}-\beta\right)} s_{c} \mid s_{i}, s_{p}\right) \\
= & \Phi\left(\frac{-\left(1+(1+k) \frac{\left(\tau_{c}+\beta\right)}{\left(\tau_{T}-\beta\right)}\right) \frac{\tau_{s}}{\tau_{s}+\tau_{p}} s_{i}-\left(\left((1+k) \frac{\left(\tau_{c}+\beta\right)}{\left(\tau_{T}-\beta\right)}+1\right) \frac{\tau_{p}}{\tau_{s}+\tau_{p}}+k\right) s_{p}}{\sqrt{\left((1+k) \frac{\left(\tau_{c}+\beta\right)}{\left(\tau_{T}-\beta\right)}+1\right)^{2} \frac{1}{\tau_{s}+\tau_{p}}+\left((1+k) \frac{\left(\tau_{c}+\beta\right)}{\left(\tau_{T}-\beta\right)} \sigma_{c}\right)^{2}}}\right)
\end{aligned}
$$

For the proposed linear strategies to be best response, the following equation must hold for a speculator at the cutoff for all $s_{p}$ :

$$
c=\Phi\left(\frac{-\left((1+k) \frac{\left(\tau_{c}+\beta\right)}{\left(\tau_{T}-\beta\right)}+1\right) \frac{\tau_{s}}{\tau_{s}+\tau_{p}} s(0)-\left(\left((1+k) \frac{\left(\tau_{c}+\beta\right)}{\left(\tau_{T}-\beta\right)}+1\right)\left(\frac{\tau_{p}}{\tau_{s}+\tau_{p}}-\frac{\tau_{s}}{\tau_{s}+\tau_{p}} k\right)+k\right) s_{p}}{\sqrt{\left((1+k) \frac{\left(\tau_{c}+\beta\right)}{\left(\tau_{T}-\beta\right)}+1\right)^{2} \frac{1}{\tau_{s}+\tau_{p}}+\left((1+k) \frac{\left(\tau_{c}+\beta\right)}{\left(\tau_{T}-\beta\right)} \sigma_{c}\right)^{2}}}\right) .
$$

Thus for the linear strategy to be a best response, the coefficient of $s_{p}$ must be zero. In other words, $k$ must satisfy:

$$
-\frac{\tau_{s}}{\tau_{s}+\tau_{p}} k+\frac{\tau_{p}}{\tau_{s}+\tau_{p}}+\frac{k}{(1+k) \frac{\left(\tau_{c}+\beta\right)}{\left(\tau_{T}-\beta\right)}+1}=0
$$

Substituting for $\tau_{T}$ and rearranging the above equation we obtain:

$$
\frac{-\beta \tau_{s}-\tau_{c} \tau_{s}}{\tau_{p}} k^{3}+k^{2}\left(\tau_{p}+\tau_{c}\right)+2 k \tau_{p}+\tau_{p}=0 .
$$

For $\beta>0$ close to zero the above equation has a unique positive root and

$$
\frac{\partial k}{\partial \beta}=-3 \frac{\tau_{s} k}{\beta \tau_{s}+\tau_{c} \tau_{s}}<0 .
$$

Furthermore,

$$
\begin{aligned}
\frac{\partial \tau_{T}}{\partial \beta} & =2 \tau_{p}\left(\frac{(1+k)}{k^{2}}-\frac{(1+k)^{2}}{k^{3}}\right) \frac{\partial k}{\partial \beta}=-6 \tau_{p}\left(\frac{(1+k)}{k^{2}}-\frac{(1+k)^{2}}{k^{3}}\right) \frac{\tau_{s} k}{\beta \tau_{s}+\tau_{c} \tau_{s}} \\
& =6 \tau_{p}\left(\frac{1+k}{k^{2}}\right) \frac{\tau_{s}}{\beta \tau_{s}+\tau_{c} \tau_{s}}>0 .
\end{aligned}
$$

Thus increasing $\beta$ always leads to a more informative attack.

Next, we compute the ex-ante probability of abandoning the status quo for a given $\theta$ :

$$
\begin{aligned}
& \operatorname{Pr}\left(T \geq \frac{1}{\sigma_{s}} s(0)+\frac{(1+k)}{\sigma_{s}} \frac{\left(\tau_{c}+\beta\right)}{\left(\tau_{T}-\beta\right)} s_{c} \mid \theta\right) \\
= & \Phi\left(-\frac{1}{\sigma_{c}} \frac{\left(1+\frac{\left(\tau_{T}-\beta\right)}{\left(\tau_{c}+\beta\right)}\right)}{\sqrt{1+\frac{\tau_{c}\left(\tau_{T}-\beta\right)^{2}}{\tau_{T}\left(\tau_{c}+\beta\right)^{2}}}} \theta\right)=\Phi\left(-\frac{1}{\sigma_{c}}\left(\frac{\tau_{c} \tau_{T}+\tau_{T}^{2}}{\tau_{c} \tau_{T}+\beta^{2}}\right)^{\frac{1}{2}} \theta\right) .
\end{aligned}
$$


Moreover,

$$
\begin{aligned}
\frac{\partial}{\partial \beta}\left(\frac{\tau_{c} \tau_{T}+\tau_{T}^{2}}{\tau_{c} \tau_{T}+\beta^{2}}\right) & =\frac{\tau_{c} \frac{\partial \tau_{T}}{\partial \beta}+2 \tau_{T} \frac{\partial \tau_{T}}{\partial \beta}}{\tau_{c} \tau_{T}+\beta^{2}}-\frac{\tau_{c} \tau_{T}+\tau_{T}^{2}}{\left(\tau_{c} \tau_{T}+\beta^{2}\right)^{2}}\left(\tau_{c} \frac{\partial \tau_{T}}{\partial \beta}+2 \beta\right) \\
& =\frac{1}{\tau_{c} \tau_{T}+\beta^{2}}\left(\left(\frac{\tau_{c} \tau_{T}^{2}+2 \tau_{T} \beta^{2}+\tau_{c} \beta^{2}}{\tau_{c} \tau_{T}+\beta^{2}}\right) \frac{\partial \tau_{T}}{\partial \beta}-\frac{\tau_{c} \tau_{T}+\tau_{T}^{2}}{\left(\tau_{c} \tau_{T}+\beta^{2}\right)} 2 \beta\right) .
\end{aligned}
$$

Computing at $\beta=0$,

$$
\left.\frac{\partial}{\partial \beta}\left(\frac{\tau_{c} \tau_{T}+\tau_{T}^{2}}{\tau_{c} \tau_{T}+\beta^{2}}\right)\right|_{\beta=0}=\left.\frac{1}{\tau_{c}} \frac{\partial \tau_{T}}{\partial \beta}\right|_{\beta=0}>0
$$

Thus, for $\theta>0(\theta<0)$ the ex-ante probability of abandoning the status quo decreases (increases) if the central bank sets $\beta$ slightly larger than zero.

\section{PROOF OF PROPOSITION 8:}

Central bank observes both $s_{c}$ and $s_{a}$, and speculators only observe $s_{a}, s_{p}$ and $s_{i}$. We construct a linear equilibrium in which a speculator attacks if and only if $s_{i} \leq \hat{s}(0)-$ $m s_{a}-k_{t r} s_{p}$ where $m>0, k_{t r}>0$. The size of the attack from speculators given $\theta, s_{p}$ and $s_{a}$ is $A\left(\theta, s_{p}\right)=\Phi\left(\frac{\hat{s}(0)-m s_{a}-k_{t r} s_{p}-\theta}{\sigma_{s}}\right)$. The central bank observes $T\left(\theta, s_{p}\right)=\Phi^{-1}(A)$, or equivalently, it observes

$$
T=\frac{\hat{s}(0)-m s_{a}-k_{t r} s_{p}-\theta}{\sigma_{s}}
$$

which can be rewritten as:

$$
\frac{\hat{s}(0)-m s_{a}-\sigma_{s} T}{1+k_{t r}}=\theta+\frac{k_{t r} \sigma_{p} \epsilon_{p}}{1+k_{t r}} .
$$

Thus, the precision of the attack as a signal of the fundamental is

$$
\tau_{T}=\frac{\tau_{p}\left(1+k_{t r}\right)^{2}}{k_{t r}^{2}}
$$

and

$$
E\left[\theta \mid T, s_{c}, \epsilon_{a}\right]=\frac{\tau_{T}}{\tau_{T}+\tau_{c}}\left(\frac{\hat{s}(0)-m\left(s_{c}+\sigma_{a} \epsilon_{a}\right)-\sigma_{s} T}{1+k_{t r}}\right)+\frac{\tau_{c}}{\tau_{T}+\tau_{c}} s_{c} .
$$

This implies the status quo is abandoned if and only if

$$
T \geq \frac{\hat{s}(0)}{\sigma_{s}}+\frac{1}{\sigma_{s}}\left(\frac{\tau_{c}}{\tau_{T}}\left(1+k_{t r}\right)-m\right) s_{c}-\frac{1}{\sigma_{s}} m \sigma_{a} \epsilon_{a} .
$$

The precision of $s_{a}$ as a signal for $\theta$ is

$$
\tau_{a}=\frac{1}{\sigma_{c}^{2}+\sigma_{a}^{2}} .
$$


Hence, for a speculator, $\theta$ is distributed with mean $\frac{\tau_{s}}{\tau_{s}+\tau_{p}+\tau_{a}} s_{i}+\frac{\tau_{p}}{\tau_{s}+\tau_{p}+\tau_{a}} s_{p}+\frac{\tau_{a}}{\tau_{s}+\tau_{p}+\tau_{a}} s_{a}$ and precision $\tau_{s}+\tau_{p}+\tau_{a}$. The posterior belief of the regime change for a speculator with signals $s_{i}, s_{p}$ and $s_{a}$ is expressed as follows:

$$
\begin{aligned}
& \operatorname{Pr}\left(T \geq \frac{\hat{s}(0)}{\sigma_{s}}+\frac{1}{\sigma_{s}}\left(\frac{\tau_{c}}{\tau_{T}}\left(1+k_{t r}\right)-m\right) s_{c}-\frac{1}{\sigma_{s}} m \sigma_{a} \epsilon_{a} \mid s_{i}, s_{p}, s_{a}\right) \\
= & \Phi\left(\frac{-m s_{a}-k_{t r} s_{p}-\left(1+\left(\frac{\tau_{c}}{\tau_{T}}\left(1+k_{t r}\right)-m\right)\right)\left(\frac{\tau_{s}}{\tau_{s}+\tau_{p}+\tau_{a}} s_{i}+\frac{\tau_{p}}{\tau_{s}+\tau_{p}+\tau_{a}} s_{p}+\frac{\tau_{a}}{\tau_{s}+\tau_{p}+\tau_{a}} s_{a}\right)}{\sqrt{\left(1+\left(\frac{\tau_{c}}{\tau_{T}}\left(1+k_{t r}\right)-m\right)\right)^{2} \frac{1}{\tau_{s}+\tau_{p}+\tau_{a}}+\left(\frac{\tau_{c}}{\tau_{T}}\left(1+k_{t r}\right)-m\right)^{2} \sigma_{c}^{2}+m^{2} \sigma_{a}^{2}}}\right)
\end{aligned}
$$

The agent with signal $\hat{s}(0)-m s_{a}-k_{t r} s_{p}$ must be indifferent between attacking or not:

$$
c=\Phi\left(\frac{-m s_{a}-k_{t r} s_{p}-\left(1+\left(\frac{\tau_{c}}{\tau_{T}}\left(1+k_{t r}\right)-m\right)\right)\left(\frac{\tau_{s}}{\tau_{s}+\tau_{p}+\tau_{a}}\left(\hat{s}(0)-m s_{a}-k_{t r} s_{p}\right)+\frac{\tau_{p} s_{p}+\tau_{a} s_{a}}{\tau_{s}+\tau_{p}+\tau_{a}}\right)}{\sqrt{\left(1+\left(\frac{\tau_{c}}{\tau_{T}}\left(1+k_{t r}\right)-m\right)\right)^{2} \frac{1}{\tau_{s}+\tau_{p}+\tau_{a}}+\left(\frac{\tau_{c}}{\tau_{T}}\left(1+k_{t r}\right)-m\right)^{2} \sigma_{c}^{2}+m^{2} \sigma_{a}^{2}}}\right) .
$$

Since in a linear equilibrium the coefficients on $s_{p}$ and $s_{c}$ must be zero, we obtain the following system of equations:

$$
\begin{array}{r}
-k_{t r}+\left(1+\left(\frac{\tau_{c}}{\tau_{T}}\left(1+k_{t r}\right)-m\right)\right) \frac{\tau_{s}}{\tau_{s}+\tau_{p}+\tau_{a}} k_{t r}-\left(1+\left(\frac{\tau_{c}}{\tau_{T}}\left(1+k_{t r}\right)-m\right)\right) \frac{\tau_{p}}{\tau_{s}+\tau_{p}+\tau_{a}}=0 \\
-m+\left(1+\left(\frac{\tau_{c}}{\tau_{T}}\left(1+k_{t r}\right)-m\right)\right) \frac{\tau_{s}}{\tau_{s}+\tau_{p}+\tau_{a}} m-\left(1+\left(\frac{\tau_{c}}{\tau_{T}}\left(1+k_{t r}\right)-m\right)\right) \frac{\tau_{a}}{\tau_{s}+\tau_{p}+\tau_{a}}=0
\end{array}
$$

To solve for $k_{t r}$ and $m$, by rearranging the two equations and take the ratio of both sides, we obtain

$$
m=\frac{\tau_{a}}{\tau_{p}} k_{t r}
$$

Substituting this into the first equation gives:

$$
\tau_{s}\left(\frac{\tau_{c}-\tau_{a}}{\tau_{p}}\right) k_{t r}^{3}+\left(\tau_{s}\left(\frac{\tau_{p}-\tau_{a}}{\tau_{p}}\right)-\tau_{s}-\tau_{p}-\tau_{c}\right) k_{t r}^{2}-2 \tau_{p} k_{t r}-\tau_{p}=0 .
$$

Notice that this equation boils down to the cubic equation we had before if we set $\tau_{a}=0$. Next we compute the discriminant:

$$
\Delta=\frac{4}{\tau_{p}^{2}}\left(\tau_{p} \tau_{c}+\tau_{a} \tau_{s}\right)\left(\tau_{p}^{2}+\tau_{c} \tau_{p}+\tau_{a} \tau_{s}\right)^{2}+\tau_{s}\left(\tau_{c}-\tau_{a}\right)\left(4 \tau_{p}^{2}+36 \tau_{c} \tau_{p}+9 \tau_{a} \tau_{s}+27 \tau_{c} \tau_{s}\right)>0 .
$$

Thus, there is a unique linear equilibrium. Moreover, in this equilibrium $k_{t r}$ and $m$ are both positive. To compare the weight $k_{t r}$ with the equilibrium weight $k$, that we obtained in Proposition 1, we evaluate equation (A1) at $k$ :

$\tau_{s}\left(\frac{\tau_{c}-\tau_{a}}{\tau_{p}}\right) k^{3}+\left(\tau_{s}\left(\frac{\tau_{p}-\tau_{a}}{\tau_{p}}\right)-\tau_{s}-\tau_{p}-\tau_{c}\right) k^{2}-2 \tau_{p} k-\tau_{p}=-\left(\frac{\tau_{s} \tau_{a}}{\tau_{p}}\right)\left(k^{3}+k^{2}\right)<0$.

This means that $k_{t r}>k$ 


\section{Appendix B}

\section{Common Signal about Noise Trading}

We now demonstrate that the central bank's policy decision is less efficient than the benchmark case even when speculators receive a common signal that is not about the fundamental. To illustrate this point suppose that there is some noise demand for the currency. Specifically, we assume that the central bank observes a noisy signal of the size of the attack from informed speculators, $T=\Phi^{-1}(A)+\sigma_{m} \epsilon_{m}$, where $A$ is the size of the aggregate attack from speculators, and the noise component of this signal, $\sigma_{m} \epsilon_{m}$, is normally distributed with a mean of zero and standard deviation $\sigma_{m} \cdot{ }^{13}$

Speculators' strategies are now functions of their private signals and the commonly observed noise level. Otherwise, equilibrium is defined analogously to the one in Definition 1. In this case, it is possible for speculators to coordinate on the level of noise demand and "fool" the central bank. The assumption on speculators' informational advantage about the noise trading level is motivated by the fact that although individual speculators may not know about the fundamental more than the central bank, they understand the institutional details or the workings of the currency market better. Under this assumption, we show that currency speculators trade more aggressively on their information when the noise component of currency attack is of a high level. As a result, the central bank cannot differentiate between a high noise attack or a low fundamental, which implies there might be occasions where the central bank on average abandons the status quo too often. We first state the result under these circumstances.

Proposition B1 There is a unique equilibrium where the speculators' threshold strategies are linear in $\epsilon_{m}$ such that

$$
g(s)= \begin{cases}1 & \text { if } \quad s_{i} \leq \bar{s}\left(\epsilon_{m}\right) \\ 0 & \text { if } \quad s_{i}>\bar{s}\left(\epsilon_{m}\right)\end{cases}
$$

and $\delta\left(T, s_{c}\right)=\left\{\begin{array}{ll}1 & \text { if } \quad T \leq \bar{T}\left(s_{c}\right) \\ 0 & \text { if } \quad T>\bar{T}\left(s_{c}\right)\end{array}\right.$, where $\bar{s}\left(\epsilon_{m}\right)=\bar{s}(0)+\bar{k} \epsilon_{m}$ where $\bar{k}>0$ is the unique real root of the cubic equation:

$$
\bar{k}^{3}+2 \sigma_{s} \sigma_{m} \bar{k}^{2}+\left(\sigma_{s} \sigma_{m}\right)^{2} \bar{k}-\sigma_{c}^{2} \sigma_{s} \sigma_{m}=0 .
$$

\footnotetext{
${ }^{13}$ This specification is introduced by Dasgupta (2005).
} 
$\bar{s}(0)$ satisfies

$$
c=\Phi\left(\frac{-\left(1+\frac{\tau_{c}}{\bar{\tau}_{T}}\right) \bar{s}(0)}{\sqrt{\left(1+\frac{\tau_{c}}{\bar{\tau}_{T}}\right)^{2} \sigma_{s}^{2}+\left(\frac{\tau_{c}}{\bar{\tau}_{T}}\right)^{2} \sigma_{c}^{2}}}\right)
$$

and

$$
\bar{T}\left(s_{c}\right)=\frac{1}{\sigma_{s}}\left[\bar{s}(0)+\frac{\left(\bar{k}+\sigma_{s} \sigma_{m}\right)^{2}}{\sigma_{c}^{2}} s_{c}\right],
$$

where $\bar{\tau}_{T}=\frac{1}{\left(\bar{k}+\sigma_{s} \sigma_{m}\right)^{2}}$ is the precision of the attack as a signal of the fundamental.

\section{PROOF OF PROPOSITION B1:}

Suppose an agent attacks if and only if $s_{i} \leq \bar{s}\left(\epsilon_{m}\right)=\bar{s}(0)+\bar{k} \epsilon_{m}$. The size of the attack from speculators is $A\left(\theta, \epsilon_{m}\right)=\Phi\left(\frac{\bar{s}\left(\epsilon_{m}\right)-\theta}{\sigma_{s}}\right)$. The central bank observes $T\left(\theta, \epsilon_{m}\right)=\Phi^{-1}(A)+\sigma_{m} \epsilon_{m}$, or equivalently, it observes

$$
\begin{aligned}
T & =\frac{\bar{s}(0)+\bar{k} \epsilon_{m}-\theta}{\sigma_{s}}+\sigma_{m} \epsilon_{m}, \\
\bar{s}(0)-\sigma_{s} T & =\theta-\left(\bar{k}+\sigma_{s} \sigma_{m}\right) \epsilon_{m},
\end{aligned}
$$

and

$$
E\left[\theta \mid T, s_{c}\right]=\frac{\bar{\tau}_{T}}{\bar{\tau}_{T}+\tau_{c}}\left(\bar{s}(0)-\sigma_{s} T\right)+\frac{\tau_{c}}{\bar{\tau}_{T}+\tau_{c}} s_{c},
$$

where $\bar{\tau}_{T}=\frac{1}{\left(\bar{k}+\sigma_{s} \sigma_{m}\right)^{2}}$. This implies the status quo is abandoned if and only if

$$
T \geq \frac{1}{\sigma_{s}}\left[\bar{s}(0)+\frac{\tau_{c}}{\bar{\tau}_{T}} s_{c}\right]=\bar{T}\left(s_{c}\right),
$$

which is Equation (B3).

The posterior belief of the regime change for a speculator with signal $s_{i}$ and $\epsilon_{m}$ is expressed as follows:

$$
\operatorname{Pr}\left(T \geq \frac{1}{\sigma_{s}}\left[\bar{s}(0)+\frac{\tau_{c}}{\bar{\tau}_{T}} s_{c}\right] \mid s_{i}, \epsilon_{m}\right)=\Phi\left(\frac{\left(\bar{k}+\sigma_{s} \sigma_{m}\right) \epsilon_{m}-\left(1+\frac{\tau_{c}}{\bar{\tau}_{T}}\right) s_{i}}{\sqrt{\left(1+\frac{\tau_{c}}{\bar{\tau}_{T}}\right)^{2} \sigma_{s}^{2}+\left(\frac{\tau_{c}}{\bar{\tau}_{T}}\right)^{2} \sigma_{c}^{2}}}\right) .
$$

Hence, $\bar{s}\left(\epsilon_{m}\right)=\bar{s}(0)+k \epsilon_{m}$ must solve

$$
c=\Phi\left(\frac{-\left(1+\frac{\tau_{c}}{\bar{\tau}_{T}}\right) \bar{s}(0)+\left(\sigma_{s} \sigma_{m}-\bar{k} \frac{\tau_{c}}{\bar{\tau}_{T}}\right) \epsilon_{m}}{\sqrt{\left(1+\frac{\tau_{c}}{\bar{\tau}_{T}}\right)^{2} \sigma_{s}^{2}+\left(\frac{\tau_{c}}{\bar{\tau}_{T}}\right)^{2} \sigma_{c}^{2}}}\right) .
$$


To solve this equation for all $\epsilon_{m}$, the coefficient of $\epsilon_{m}$ must be zero. In other words,

$$
\sigma_{s} \sigma_{m}-\bar{k} \frac{\tau_{c}}{\bar{\tau}_{T}}=0
$$

Rearranging we find that $\bar{k}$ must solve:

$$
\bar{k}^{3}+2 \sigma_{s} \sigma_{m} \bar{k}^{2}+\left(\sigma_{s} \sigma_{m}\right)^{2} \bar{k}-\sigma_{c}^{2} \sigma_{s} \sigma_{m}=0 .
$$

To see that this equation has a unique real root we compute the discriminant $\Delta$ of the cubic equation:

$$
\Delta=4\left(\sigma_{s} \sigma_{m}\right)^{4} \frac{1}{\tau_{c}}+27\left(\frac{\sigma_{s} \sigma_{m}}{\tau_{c}}\right)^{2}>0 .
$$

Since $\Delta>0$ the equation has a unique real root. Moreover, the left-hand side of equation (B6) goes to $-\infty$ as $\bar{k}$ goes to $-\infty$ and it is negative at $\bar{k}=0$. Since the equation has a single real root, it must cross zero at some $\bar{k}>0$. Given $\bar{k}$, we obtain $\bar{s}(0)$ as the solution to Equation (B2).

In other words, the equilibrium strategy for the central bank is to abandon the exchange regime if and only if the observed signal of aggregate attack, $T$, is greater than or equal to the threshold, $\bar{T}\left(s_{c}\right)$, a function of the central bank's private signal, $s_{c}$. The equilibrium strategy for a speculator who receives a signal, $s$, is to attack if and only if $s$ falls below a threshold value, $\bar{s}\left(\epsilon_{m}\right)$, which is a linear increasing function of the noise trading level in the currency market.

Proposition B2 The weight $\bar{k}$ put by speculators on $\epsilon_{m}$ in the unique linear threshold equilibrium characterized by Proposition $B 1$ is greater than the weight $\bar{k}_{B M}$ that would be put on $\epsilon_{m}$ in a game where the central bank does not attempt to get information about $\theta$ from the size of the attack.

The proof is straightforward since $\bar{k}$ is strictly positive and if the central bank does not infer the fundamental from $T, \bar{k}_{B M}=0$.

Empirically, it has been difficult to explain the fact that speculative attacks tend to be contagious even though the fundamentals of the countries under attack are quite different. Our finding in this section offers an explanation. Since the noise trading level tends to be high across countries during the periods of market upheavals such as the ERM debacles (1992), the Asian crisis (1997), and the Russian default (1998), currency speculators, knowing so, coordinate their attacks across countries around these times. Given this finding, central 
banks should adjust their behavior during contagious speculative attacks - they should rely less on market information to make policy decisions.

\section{Appendix C}

\section{Speculators Observe Fundamental Perfectly}

We begin by defining equilibrium in this setting. Let $A(\theta)$ denote the size of the aggregate attack given $\theta, g(\theta)$ the action of an agent given $\theta$, and $\delta\left(A, s_{c}\right)$ the action of the central bank as a function of the size of the attack and its signal. Furthermore, let $\nu\left(\theta \mid A, s_{c}\right)$ denote the posterior belief by the central bank conditional on $A$ and $s_{c}$.

Definition C1 An equilibrium consists of a mapping A from the fundamental to the size of the attack, a strategy for the central bank, $\delta\left(A, s_{c}\right)$, a symmetric strategy for the agents, $g(\theta)$ and a probability measure, $\nu\left(\cdot \mid A, s_{c}\right)$, such that

$$
\begin{aligned}
& \delta\left(A, s_{c}\right) \in \operatorname{argmax}_{\delta \in\{0,1\}} \int_{-\infty}^{\infty} \delta \theta d \nu\left(\theta \mid A, s_{c}\right), \\
& g(\theta) \in \operatorname{argmax}_{a \in\{0,1\}} a \cdot\left[\int_{-\infty}^{\infty} \mathbf{1}_{\left[\delta\left(A(\theta), \theta+\sigma_{c} \epsilon_{c}\right)=0\right]} d \phi\left(\epsilon_{c}\right)-c\right] \\
& \nu\left(\theta \mid A, s_{c}\right) \text { is obtained using Bayes' rule for any } A, s_{c}, \\
& A(\theta)=1 \text { if } g(\theta)=1 \text { and } A(\theta)=0 \text { otherwise. }
\end{aligned}
$$

Since speculators have information that is valuable to the central bank, in equilibrium, the central bank would make its policy decision based on the information revealed through speculators' actions as well as its own noisy private signal. When the fundamental is common knowledge among them, speculators may coordinate in various ways leading to different inferences by the central bank, resulting in multiple equilibria. In particular, speculators may be able to convince the central bank to abandon the fixed exchange rate regime even when it is not optimal for the central bank to do so. Our next proposition shows that multiplicity may arise when the speculators follow symmetric cut-off strategies.

Proposition C1 There exists $\theta^{*}>0$ such that for all $\bar{\theta} \in\left[0, \theta^{*}\right]$ there is an equilibrium where $g(\theta)=1$ if $\theta \leq \bar{\theta}$ and $g(\theta)=0$ if $\theta>\bar{\theta}$.

\section{PROOF OF PROPOSITION C1:}

Suppose $g(\theta)=1$ if $\theta \leq \bar{\theta}$ and $g(\theta)=0$ if $\theta>\bar{\theta}$. Hence, the expectation of $\theta$ conditional on 
observing $A=1$ and $s_{c}$ is

$$
E\left[\theta \mid \theta \leq \bar{\theta}, s_{c}\right]=\frac{\int_{-\infty}^{\bar{\theta}} \theta \phi\left(\frac{\theta-s_{c}}{\sigma_{c}}\right) d \theta}{\Phi\left(\frac{\bar{\theta}-s_{c}}{\sigma_{c}}\right)} .
$$

Next, we show that $E\left[\theta \mid \theta \leq \bar{\theta}, s_{c}\right]$ is increasing in $s_{c}$. To see this, note that $E\left[\theta \mid \theta \leq \bar{\theta}, s_{c}+\Delta\right]=$ $\Delta-E\left[\theta \mid \theta \leq \bar{\theta}-\Delta, s_{c}\right]$. Subtracting $E\left[\theta \mid \theta \leq \bar{\theta}, s_{c}\right]$ from both sides, dividing by $\Delta$ and letting $\Delta$ go to zero, we obtain:

$$
\frac{\partial E\left[\theta \mid \theta \leq \bar{\theta}, s_{c}\right]}{\partial s_{c}}=1-\frac{\partial E\left[\theta \mid \theta \leq \bar{\theta}, s_{c}\right]}{\partial \bar{\theta}} .
$$

By Proposition 1 in Burdett (1996), we know that $\frac{\partial E\left[\theta \mid \theta \leq \bar{\theta}, s_{c}\right]}{\partial \bar{\theta}} \in[0,1]$. Thus, $\frac{\partial E\left[\theta \mid \theta \leq \bar{\theta}, s_{c}\right]}{\partial s_{c}} \geq 0$. Suppose $\bar{\theta} \geq 0$. Since $\frac{\partial E\left[\theta \mid \theta \leq \bar{\theta}, s_{c}\right]}{\partial s_{c}} \geq 0$, there exists a $\hat{s}_{c}(\bar{\theta})>0$ (possibly infinite) such that

$$
E\left[\theta \mid \theta \leq \bar{\theta}, s_{c}\right]=\left\{\begin{array}{lll}
\geq 0 & \text { if } & s \geq \hat{s}_{c}(\bar{\theta}) \\
<0 & \text { if } \quad s<\hat{s}_{c}(\bar{\theta})
\end{array} .\right.
$$

Therefore, the central bank's strategy is

$$
\delta\left(A, s_{c}\right)=\left\{\begin{array}{cc}
1 & \text { if } \quad A=1 \text { and } s_{c}>\hat{s}_{c}(\bar{\theta}) \text { or } A=0 \\
0 & \text { o.w. }
\end{array} .\right.
$$

Moreover, $\hat{s}_{c}(\bar{\theta})$ is decreasing in $\bar{\theta}$.

Given the central bank's strategy and given the strategy of the other speculators, it is optimal for any speculator to set $g(\theta)=0$ if $\theta>\bar{\theta}$. Now, fix some $\theta \leq \bar{\theta}$. We want to show that if $\bar{\theta}<\theta^{*}$ for some $\theta^{*}>0$ then it is optimal for the speculators to set $g(\theta)=1$. Since in this case, $A=1$ and the probability that the central bank abandons the regime is $\Phi\left(\frac{\hat{s}_{c}(\bar{\theta})-\theta}{\sigma_{c}}\right)$. Note that this probability is decreasing in $\theta$, so if it is optimal for speculators to attack at $\bar{\theta}$, it is also optimal to attack at all smaller $\theta$. Now note that $\Phi\left(\frac{\hat{s}_{c}(\bar{\theta})-\bar{\theta}}{\sigma_{c}}\right)$ is one at $\bar{\theta}=0$, is continuous and decreases as $\bar{\theta}$ increases. Therefore, there is a threshold $\theta^{*}>0$ such that $\Phi\left(\frac{\hat{s}_{c}(\bar{\theta})-\bar{\theta}}{\sigma_{c}}\right)-c \gtreqless 0$ if $\bar{\theta} \lesseqgtr \theta^{*}$. Thus it is optimal to attack for the speculators if $\theta \leq \bar{\theta}$ where $0 \leq \bar{\theta} \leq \theta^{*}$. This proves the proposition.

In other words, this proposition shows that there are multiple equilibria in which the speculators follow a cut-off strategy: They attack the currency regime when their signal is below $\bar{\theta}$ and do not attack otherwise. The cut-off value could be any $\theta$ between $\left[0, \theta^{*}\right]$. This means that the central bank may devalue when the fundamental, $\theta$, is positive, which is not first-best optimal for the central bank. 


\section{References}

Angeletos, George-Marios, Christian Hellwig, and Alessandro Pavan, 2007, Dynamic global games of regime change: Learning, multiplicity and timing of attacks, Econometrica 75, $711-756$.

Angeletos, George-Marios, Guido Lorenzoni, and Alessandro Pavan, 2007, Wall Street and Silicon Valley: A delicate interaction, MIT working paper.

Angeletos, George-Marios, and Alessandro Pavan, 2007, Efficient use of information and social value of information, Econometrica 75, 1103-1142.

Angeletos, George-Marios, and Ivan Werning, 2006, Crises and prices - information aggregateion, multiplicity and volatility, American Economic Review 96, 1720-36.

Baker, Malcolm, Jeremy Stein, and Jeffrey Wurgler, 2003, When does the market matter? Stock prices and the investment of equity-dependent firms, Quarterly Journal of Economics 118, 969-1006.

Boot, Arnoud, and Anjan Thakor, 1997, Financial system architecture, Review of Financial Studies 10, 693-733.

Bru, Lluis, and Xavier Vives, 2002, Informational externalities, herding and incentives, Journal of Institutional and Theoretical Economics 158, 91-105.

Burnside, Craig, Martin Eichenbaum, and Sergio Rebelo, 2001, Prospective deficits and the Asian currency crisis, Journal of Political Economy 109, 1155-1197.

Chen, Qi, Itay Goldstein, and Wei Jiang, 2007, Price informativeness and investment sensitivity to stock price, Review of Finacial Studies 20, 619-650.

Danielsson, Jon, and Burak Saltoglu, 2003, Anatomy of a market crash: A market microstructure analysis of the Turkish overnight liquidity crisis, London School of Econonomics working paper.

Dasgupta, Amil, 2005, Coordination and delay in global games, Journal of Economic Theory forthcoming. 
Dow, James, Itay Goldstein, and Alexander Guembel, 2007, Incentives for information production in markets where prices affect real investment, Working Paper.

Dow, James, and Gary Gorton, 1997, Stock market efficiency and economic efficiency: Is there a connection? Journal of Finance 52, 1087-1129.

Drazen, Allan, 2000, Interest-rate and borrowing defense against speculative attack, Carnegie-Rocehster Conference Series on Public Policy 53, 303-348.

Easley, David, and Maureen O'Hara, 1987, Price, trade size, and information in securities markets, Journal of Financial Economics 19, 69-90.

Evans, Martin D. D., and Richard K. Lyons, 2002, Order flow and exchange rate dynamics, Journal of Political Economy 19, 170-180.

Fishman, Mike J., and Kathleen M. Hagerty, 1992, Insider trading and the efficiency of stock prices, RAND Journal of Economics 23, 106-122.

Flood, Robert, and Peter Garber, 1984, Collapsing exchange-rate regimes: Some linear examples, Journal of International Economics 17, 1-13.

Froot, Kenneth A., David Scharfstein, and Jeremy Stein, 1992, Herd on the street: Informational inefficiencies in a market with short-term speculation, Journal of Finance 47, $1461-1484$.

Fulghieri, Paolo, and Dmitry Lukin, 2001, Information production, dilution costs, and optimal security design, Journal of Financial Economics 61, 3-42.

Goldstein, Itay, and Alexander Guembel, 2007, Manipulation and the allocational role of prices, Review of Economic Studies forthcoming.

Heinemann, Frank, and Camille Cornand, 2004, Optimal degree of public information dissemination, CESifo working paper 1354.

Hellwig, Christian, 2005, Heterogeneous information and the benefits of transparency, UCLA working paper.

, Arijit Mukherji, and Aleh Tsyvinski, 2006, Self-fulfilling currency crises: The role of interest rates, American Economic Review 96, 1769-87. 
Hirshleifer, David, Avanidhar Subrahmanyam, and Sheridan Titman, 1994, Security analysis and trading patterns when some investors receive information before others, Journal of Finance 49, 1665-1698.

Ito, Takatoshi, Richard K. Lyons, and Michael T. Melvin, 1998, Is there private information in the foreign exchange market? The Tokyo experiment, Journal of Finance 53, 1111-1130.

Khanna, Naveen, Steve L. Slezak, and Michael H. Bradley, 1994, Insider trading, outside search and resource allocation: Why firms and society may disagree on insider trading restrictions, Review of Financial Studies 7, 575-608.

Khanna, Naveen, and Ramana Sonti, 2004, Value creating stock manipulation: Feedback effect of stock prices on firm value, Journal of Financial Markets 7, 237-270.

Krugman, Paul, 1979, A model of self-fulfilling balance of payments crises, Journal of Money, Credit, and Banking 11, 311-25.

Leland, Hayne, 1992, Insider trading: Should it be prohibited? Journal of Political Economy $100,859-887$.

Luo, Yuanzhi, 2005, Do insiders learn from outsiders? Evidence from mergers and acquisitions, Journal of Finance 60, 1951-1972.

Lyons, Richard K., 2001, The Microstructure Approach to Exchange Rates (MIT Press: Cambridge, MA).

Morris, Stephen, and Hyun Song Shin, 1998, Unique equilibrium in a model of self-fulfilling currency attacks, American Economic Review 88, 587-597.

— , 2002, The social value of public information, American Economic Review 92, 15211534.

— 2005, Central bank transparency and the signal value of prices, Brookings Papers on Economic Activity.

Obstfeld, Maurice, 1996, Models of currency crises with self-fulfilling features, European Economic Review 40, 1037-48.

O'Hara, Maureen, 1995, Market Microstructure Theory (Blackwell Publishers: Cambridge, MA). 
Ozdenoren, Emre, and Kathy Yuan, 2007, Feedback effects and asset prices, Journal of Finance forthcoming.

Piazzesi, Monika, 2005, Bond yields and the Federal Reserve, Journal of Political Economy $113,311-344$.

Rebelo, Sergio, 2000, Comment on "Interest rate and borrowing defense against speculative attack" by Allan Drazen, Northwestern University working paper.

Roll, Richard, 1984, Orange juice and weather, American Economic Review 74, 861-880.

Salant, Stephen W., and Dale W. Henderson, 1978, Market anticipations of government policies and the price of gold, Journal of Political Economy 86, 627-648.

Subrahmanyam, Avanidhar, and Sheridan Titman, 1999, The going-public decision and the development of financial markets, Journal of Finance 54, 1045-1082.

Svensson, Lars, 2005, The social value of public information: Morris and Shin (2002) is actually pro transparency, not con, American Economic Review 96, 448-452.

Veldkamp, Laura, 2006a, Information markets and the comovement of asset prices, Review of Economic Studies 73, 823-845.

— , 2006b, Media frenzies in markets for financial information, American Economic Review 96, 577-601.

Wolfers, Justin, and Eric Zitzewitz, 2004, Prediction markets, Journal of Economic Perspectives 18, 107-126.

Woodford, Michael, 2005, Central bank communication and policy effectiveness, 2005 Symposium of the Federal Reserve Bank of Kansas City at Jacksons Hole, The Greenspan Era: Lessons for the Future. 